Preprint typeset in JHEP style - HYPER VERSION

KIAS-P09054

\title{
Schrödinger invariant solutions of M-theory with Enhanced Supersymmetry
}

\author{
Jaehoon Jeong ${ }^{a}$, Hee-Cheol $\mathrm{Kim}^{b}$, Sangmin Lee ${ }^{c, d}$, Eoin Ó Colgáin ${ }^{d}$, Hossein \\ Yavartanoo $^{d}$ \\ ${ }^{a}$ Department of Physics, College of Science, Yonsei University, Seoul 120-749, Korea \\ ${ }^{b}$ Department of Physics and Astronomy, Seoul National University, Seoul 151-747, Korea \\ ${ }^{c}$ Center for Quantum Space-time, Sogang University, Seoul 121-742, Korea \\ ${ }^{d}$ Korea Institute for Advanced Study, Seoul 130-722, Korea
}

\begin{abstract}
We find the most general solution of 11-dimensional supergravity compatible with $\mathcal{N}=2$ super-Schrödinger symmetry with six supercharges and $\operatorname{SU}(2) \times S U(2) \times$ $U(1) \times \mathbb{Z}_{2}$ global symmetry. It can be viewed as a one-parameter extension of a recently constructed solution by Ooguri and Park. Our original motivation was to find the gravity dual of the non-relativistic ABJM theory. But, our analysis shows that no such solution exists within the reach of our assumptions. We discuss possible reasons for the non-existence of the desired solution. We also uplift a super-Schrödinger solution in IIB supergravity of Donos and Gauntlett to 11-dimension and comment on its properties.
\end{abstract}

KEYWORDS: Schrödinger symmetry, Non-relativistic holography, Supergravity. 


\section{Contents}

1. Introduction

2 Motivation and Setup 目

2.1 Non-relativistic ABJM theory and BW/LLM solution B

2.2 Super-Schrödinger symmetry 6

2.3 Ansatz 9

3. Solution and a Sketch of the Computation 11

3.1 Methods 11

3.2 Killing spinor equations: summary

3.3 Solution 13

4. Details of the Computation 15

4.1 Killing spinor equations 15

4.2 Spinorial Lie derivatives 17

4.3 Kinematical supercharges and null Killing spinor 18

4.4 Dynamical supercharges and time-like Killing spinor 23

5. $S^{2} \times T^{2}$ solution 27

6. Discussion 30

A. Notations and Conventions 32

B. Bena-Warner/Lin-Lunin-Maldacena solution 32

\section{Introduction}

The AdS/CFT correspondence in its various guises is now more than a decade old. From its original incarnation connecting $\mathcal{N}=4$ super Yang-Mills theory and near horizon geometry of D3-branes, via less supersymmetric models closer in nature to $\mathrm{QCD}$, the conjectured AdS/CFT has passed numerous non-trivial hurdles, thus ensuring its place as one of the cornerstones of the string theory literature. 
Emboldened by such successes, physicists recently have shifted tack to applying the AdS/CFT to model conformal quantum mechanical condensed matter systems with nonrelativistic (NR) symmetry. In this setting, [1,2] initiated a flurry of excitement in a NR version of the AdS/CFT by proposing a gravity background whose isometry group is the so-called Schrödinger group with dynamical exponent $z$. We will focus on the $z=2$ case, where the Schrödinger group consists of space and time translations, Galilean boosts, a scale transformation and a special conformal transformation.

To study whether and how the NR-AdS/CFT works, it would be desirable to have a concrete example of a dual pair with a large amount of supersymmetry, i.e., a NR analog of $\mathcal{N}=4$ super-Yang-Mills and $A d S_{5} \times S^{5}$. A notable example in this regard is the "nonrelativistic mass deformed ABJM theory" (NR-ABJM) constructed recently in $[3,4]$ based on the $(2+1)$-dimensional $\mathcal{N}=6$ Chern-Simons matter theory of Aharony, Bergman, Jafferis and Maldacena [5]. The NR-ABJM theory has global symmetry group $U(1)_{B} \times$ $S U(2)_{1} \times S U(2)_{2} \times U(1)_{R} \times \mathbb{Z}_{2}$ and 14 supersymmetries.

The original ABJM theory at Chern-Simons level $(k,-k)$ describes multiple M2-branes probing the orbifold $\mathbb{C}^{4} / \mathbb{Z}_{k}$ in the transverse direction; the gravity dual is $A d S_{4} \times S^{7} / \mathbb{Z}_{k}{ }^{1}$ One may turn on an anti-self-dual four-form flux in $\mathrm{C}^{4}$, which polarizes M2-branes into M5-branes [6-8]. This corresponds to the mass deformation of the ABJM theory $[9,10]$ with the most symmetric (classical) vacuum having the global symmetry $S U(2) \times S U(2) \times$ $U(1) \times \mathbb{Z}_{2}$ and 12 Poincaré supersymmetries.

In the course of taking the non-relativistic limit, the internal symmetry of the vacuum remains unchanged, while the space-time symmetry mutates into the Schrödinger symmetry. At the same time, the supersymmetry is enhanced from 12 to 14 supercharges. The latter may be divided into the sum of 2 dynamical, 2 kinematical and 2 conformal supercharges constituting the $\mathcal{N}=2$ super-Schrödinger algebra [11-13], as well as 8 additional "spectator" supercharges.

If the classical analysis of the field theory vacuum structure may be transplanted directly to the the supergravity setting, the gravity dual of the NR-ABJM theory could simply be found by taking a suitable "non-relativistic limit" of the solution of $[7,8]$. However, as we will discuss below, there are some conceptual and technical difficulties for such an operation, which leads us to pursue an alternative approach.

We start by constructing an ansatz for 11-dimensional supergravity that is compatible with all the global symmetry and Schrödinger symmetry of the NR-ABJM theory, and proceed to analyze the Killing spinor equations. We succeed in finding the most general solution with 6 supercharges forming the $\mathcal{N}=2$ super-Schrödinger algebra. The solution takes a simple, explicit form and includes two free parameters $(b, c)$. Setting $b=0$, we recover the one-parameter family of solutions previously found by Ooguri and Park [14], where the result was obtained by deforming some known $\mathcal{N}=1 A d S_{5}$ solutions in $\mathrm{M}$ theory $[15]^{2}$.

\footnotetext{
${ }^{1}$ We will set $k=1$ for most of our discussion, although generalization for arbitrary $k$ is straightforward.

${ }^{2}$ Earlier work on non-relativistic deformations of this supersymmetric family appeared in [16].
} 
However, bearing in mind the original goal of realizing the 8 additional supercharges, we are forced to conclude that, within the reach of our assumptions, the desired solution does not exist. We will list several possible explanations for the failure, but the discussion will not be conclusive. ${ }^{3}$

We use standard methods for solving the Killing spinor equations, namely, spinorial Lie derivatives and G-structure. The methods may be easily adapted to generate more super-Schrödinger solutions, but with the lengthy analysis involved, we confine ourselves to this one example. Instead, to illustrate how to compare with previously known $\mathcal{N}=2$ super-Schrödinger solutions [18, 19], we perform T-duality on the IIB solution of [19] to obtain a new solution in M-theory containing an $S^{2} \times T^{2}$ component in the internal space.

The rest of this paper is organized as follows. In section 2, we review some relevant features of both NR-ABJM theory and super-Schrödinger symmetry. We also exhibit our ansatz compatible with the expected global symmetries. In section 3, after a brief introduction to our methods, we present the solution, explain its main features, compare it with the solution of [14]. Section 4 contains the details of solving the Killing spinor equations. In section 5, we uplift a IIB solution of [19] to M-theory and comment on its properties. We conclude in section 6 with a discussion on possible reasons why the gravity dual of NR-ABJM does not exist within reach of our assumptions.

\section{Motivation and Setup}

\subsection{Non-relativistic ABJM theory and BW/LLM solution}

A brief review of NR-ABJM The ABJM theory is an $\mathcal{N}=6$ supersymmetric ChernSimons-matter theory with $U(N) \times U(N)$ gauge group with Chern-Simons levels $(k,-k)$. The matter fields consist of bi-fundamental scalars $\Phi^{A}$ and fermions $\Psi_{A}$, which transform under the $S U(4) \simeq S O(6) R$-symmetry group as 4 and $\overline{4}$, respectively.

The theory is dual to M-theory on $A d S_{4} \times S^{7} / \mathbb{Z}_{k}$. Regarding $S^{7}$ as a circle fibration over $\mathbb{C P}^{3}$, the $\mathbb{Z}_{k}$ acts on the the fiber. In other words, the $\mathbb{Z}_{k}$ action breaks the $S O(8)$ symmetry of $S^{7}$ to $U(1)_{B} \times S U(4)$. It is sometimes useful to take the $U(1)_{B}$ direction to be the M-theory circle and consider IIA theory on $A d S_{4} \times \mathbb{C P}^{3}$ with fluxes turned on. In the field theory the $U(1)_{B}$ generator counts the total number of bosons and fermions.

The non-relativistic ABJM theory with 14 supercharges (NR-ABJM) [3,4] can be obtained in two steps. First, one performs a mass deformation $[9,10]$ which gives the same mass to all matter fields (up to signs for fermions) and breaks the $S U(4) R$-symmetry into $S U(2)_{1} \times S U(2)_{2} \times U(1)_{R}$. Second, one takes the usual non-relativistic limit for massive fields. The Lagrangian of the resulting theory is as follows:

$$
\mathcal{L}=\frac{k}{4 \pi}\left(\mathcal{L}_{\mathrm{CS}}+\mathcal{L}_{\text {kin }}+\mathcal{L}_{\text {bos }}+\mathcal{L}_{\text {int } 1}+\mathcal{L}_{\text {int } 2}\right)
$$

\footnotetext{
${ }^{3}$ See [17] for a possibly related discussion.
} 
where

$$
\begin{aligned}
\mathcal{L}_{\mathrm{CS}}= & \epsilon^{m n p} \operatorname{tr}\left[A_{m} \partial_{n} A_{p}-\frac{2 i}{3} A_{m} A_{n} A_{p}-\tilde{A}_{m} \partial_{n} \tilde{A}_{p}+\frac{2 i}{3} \tilde{A}_{m} \tilde{A}_{n} \tilde{A}_{p}\right], \\
\mathcal{L}_{\text {kin }}= & \operatorname{tr}\left[\bar{\phi}_{A}\left(i D_{t}\right) \phi^{A}-\left(D_{i} \bar{\phi}_{A}\right)\left(D_{i} \phi^{A}\right)\right] \\
& +\operatorname{tr}\left[\bar{\psi}^{A}\left(i D_{t}\right) \psi_{A}+\bar{\psi}^{a}\left(D_{i}^{2} \psi_{a}-F_{12} \psi_{a}+\psi_{a} \bar{F}_{12}\right)+\bar{\psi}^{\dot{a}}\left(D_{i}^{2} \psi_{\dot{a}}+F_{12} \psi_{\dot{a}}-\psi_{\dot{a}} \bar{F}_{12}\right)\right], \\
\mathcal{L}_{\text {bos }}= & \frac{1}{2} \operatorname{tr}\left[\phi^{a} \bar{\phi}_{[a} \phi^{b} \bar{\phi}_{b]}-\phi^{\dot{a}} \bar{\phi}_{[\dot{a}} \phi^{\dot{b}} \bar{\phi}_{\dot{b}]}\right], \\
\mathcal{L}_{\text {int } 1}= & \frac{1}{4} \operatorname{tr}\left[\left(\bar{\phi}_{a} \phi^{a}+\bar{\phi}_{\dot{a}} \phi^{\dot{a}}\right)\left(\bar{\psi}^{b} \psi_{b}-\bar{\psi}^{\dot{b}} \psi_{\dot{b}}\right)+\left(\phi^{a} \bar{\phi}_{a}+\phi^{\dot{a}} \bar{\phi}_{\dot{a}}\right)\left(\psi_{b} \bar{\psi}^{b}-\psi_{\dot{b}} \bar{\psi}^{\dot{b}}\right)\right] \\
& +\frac{1}{2} \operatorname{tr}\left[-\phi^{a} \bar{\phi}_{b} \psi_{a} \bar{\psi}^{b}+\phi^{\dot{a}} \bar{\phi}_{\dot{b}} \psi_{\dot{a}} \bar{\psi}^{\dot{b}}-\bar{\phi}_{a} \phi^{b} \bar{\psi}^{a} \psi_{b}+\bar{\phi}_{\dot{a}} \phi^{\dot{b}} \bar{\psi}^{\dot{a}} \psi_{\dot{b}}\right], \\
\mathcal{L}_{\text {int2 }}= & -\frac{1}{2} \operatorname{tr}\left[\epsilon^{a b} \epsilon^{\dot{c} \dot{d}}\left(\bar{\phi}_{a} \psi_{b} \bar{\phi}_{\dot{c}} \psi_{\dot{d}}+\bar{\phi}_{a} \psi_{\dot{c}} \bar{\phi}_{\dot{d}} \psi_{b}\right)+\epsilon_{a b} \epsilon_{\dot{c} \dot{d}}\left(\phi^{a} \bar{\psi}^{b} \phi^{\dot{c}} \bar{\psi}^{\dot{d}}+\phi^{a} \bar{\psi}^{\dot{c}} \phi^{\dot{d}} \bar{\psi}^{b}\right)\right] .
\end{aligned}
$$

We are mainly following the notations of [3] with some minor changes. The $(a, b ; \dot{a}, \dot{b})$ indices denote doublets of $S U(2)_{1} \times S U(2)_{2}$.

The Lagrangian (2.2) is invariant under the scaling

$$
(t, x ; \phi, \psi) \rightarrow\left(\lambda^{-2} t, \lambda^{-1} x ; \lambda \phi, \lambda \psi\right) .
$$

As in the free Schrödinger field theory, this scaling symmetry can be extended to the full Schrödinger algebra which also includes a non-relativistic special conformal symmetry generator $K$.

As for the supersymmetry, all 12 Poincaré supercharges of the ABJM theory survive the mass deformation as well as the non-relativistic limit. Four of them are singlets under $S U(2)_{1} \times S U(2)_{2}$. Two of them $(Q, \bar{Q})$, which anti-commute to give the Hamiltonian $H$, are called dynamical. The other two $(q, \bar{q})$ which anti-commute to give the $U(1)_{B}$ generator are called kinematical. These supercharges transform non-trivially under the Schrödinger algebra. In particular, the commutators between $K$ and $(Q, \bar{Q})$ require that an additional pair of supercharges $(S, \bar{S})$, called conformal supercharges, should exist. These six supercharges together with the Schrödinger generators form the so-called $\mathcal{N}=2$ super-Schrödinger algebra as we will discuss in more detail below.

The remaining eight supercharges $\left\{q_{a \dot{a}}, \bar{q}^{a \dot{a}}\right\}$, which we call spectators, commute with all Schrödinger generators except the rotation, and transform in $(2,2)$ of $S U(2)_{1} \times S U(2)_{2}$. In summary, the NR-ABJM theory has the global symmetry group

$$
U(1)_{B} \times S U(2)_{1} \times S U(2)_{2} \times U(1)_{R} \times \mathbb{Z}_{2},
$$

where the $\mathbb{Z}_{2}$ interchanges the two $S U(2)$ factors, and contains 14 supercharges.

BW/LLM solution and subtleties with the NR limit The gravity dual of the ABJM theory is $\mathrm{AdS}_{4} \times S^{7} / \mathbb{Z}_{k}$. To find the gravity dual of the NR-ABJM theory, a naive approach would be to carry over the mass deformation and the non-relativistic limit to the gravity side. But, a moment's thought reveals difficulties in such an attempt. 
The gravity dual of the mass deformed theory was obtained some time ago by Bena and Warner [7] (BW) and reproduced later by Lin, Lunin and Maldacena [8] (LLM); see appendix B for a short summary of the BW/LLM solution. Bena-Warner begins with a collection of M2-branes and turns on the four-form flux in the transverse directions. The flux breaks the $S O(8) R$-symmetry to $S O(4) \times S O(4)$ and polarizes the M2-branes into M5-branes, which wrap the two three-spheres that are orbits of the $S O(4)$ groups.

There exists a gravity solution for each distinct configuration of polarized M5-branes. Remarkably, the supergravity equations boil down to a linear equation. In the language of LLM, the smooth solutions are in one-to-one correspondence with Young tableaux whose total number of boxes $N$ are the same as the number of M2-branes before polarization. It is widely believed that the polarized M5-branes correspond to "fuzzy three-sphere" configurations of the ABJM theory, although an exact match at the quantum level still remains an open problem [10].

Note that the non-relativistic limit of the mass deformed ABJM theory was taken without taking the polarization effects into account. It would correspond to a BW/LLM solution with no polarized M5-branes. Such a solution was written down in [7], but was found to exhibit a naked singularity. The LLM dictionary makes it clear that the singularity is unavoidable.

Putting the singularity problem aside for a moment, let us consider how to perform the non-relativistic limit on the gravity side. The NR-ABJM theory is non-trivial when there are non-zero number of particles, which is proportional to the eigenvalue of the $U(1)_{B}$ generator, which in turn gets identified with the central element $M$ of the Schrödinger algebra.

Recall that the $U(1)_{B}$ generator acts on the circle fiber of $S^{7}$. On the other hand, in the geometric realization of the Schrödinger algebra to be reviewed in the next subsection, $M$ is identified with a light-cone momentum. The situation is strongly reminiscent of the discrete light-cone quantization (DLCQ) procedure taken in the context of Schrödinger geometry in [20-22]. A crucial difference is that in our case the light-cone momentum is taken along a direction transverse to the M2-brane world-volume. The existence of this light-cone momentum also hinders attempts to obtain solutions via consistent truncation, as were performed in $[21,23,24]$.

In principle, one could proceed as follows. First, one modifies the BW/LLM solution by adding the particle number $M$. In the IIA picture, it amounts to turning on the flux counting the D0-brane charge. Second, one makes the standard coordinate change of the DLCQ procedure: ${ }^{4}$

$$
\begin{aligned}
& \tilde{\phi}=\phi-\alpha t, \quad \tilde{t}=t \\
\Rightarrow & \widetilde{H} \equiv i \partial_{\tilde{t}}=i \partial_{t}-\alpha\left(-i \partial_{\phi}\right) \equiv H-\alpha M, \quad \widetilde{M} \equiv-i \partial_{\tilde{\phi}}=-i \partial_{\phi} \equiv M .
\end{aligned}
$$

With a suitably chosen constant $\alpha$ and an appropriate scaling limit, the light-cone Hamiltonian is identified with the Hamiltonian of the non-relativistic theory. The gravity description is expected to be valid for a large value of $M$.

\footnotetext{
${ }^{4}$ See [14] for a closely related discussion.
} 
Coming back to the BW/LLM solution, it is conceivable that the scaling limit of the DLCQ procedure may push away the singularity of the unpolarized solution, so that the final non-relativistic solution becomes free of any singularity. Whether such a phenomenon happens could be tested only by a direct computation. Unfortunately, we are hindered by a technical difficulty; it is not clear how to turn on the $M$ momentum and obtain the fully back reacted supergravity solution, as the $U(1)_{B}$ circle is fibered non-trivially along the $\mathbb{C P}^{3}$ base.

We are thus led to an alternative approach. We will begin with the most general ansatz consistent with the symmetries of the NR-ABJM theory and look for a supergravity solution preserving the same amount of supersymmetry. Before writing down the ansatz, we review the super-Schrödinger algebra in some detail.

\subsection{Super-Schrödinger symmetry}

Bosonic algebra in arbitrary dimensions The Schrödinger algebra $\mathrm{Sch}_{d}$ contains an $S O(2,1)$ subalgebra among the time-translation $(H)$, dilatation $(D)$ and special conformal (C) generators.

$$
[D, H]=+2 H, \quad[D, C]=-2 C, \quad[H, C]=-D,
$$

as well as the $S O(d)$ subalgebra,

$$
\left[M^{i j}, M^{k l}\right]=+\delta^{j k} M^{i l}+\delta^{i l} M^{j k}-\delta^{i k} M^{j l}-\delta^{j l} M^{i k}
$$

The remaining generators are space-translations $\left(P^{i}\right)$ and Galilean boosts $\left(G^{i}\right)$. They are vectors under the $S O(d)$,

$$
\left[M^{i j}, P^{k}\right]=+\delta^{j k} P^{i}-\delta^{i k} P^{j}, \quad\left[M^{i j}, G^{k}\right]=+\delta^{j k} G^{i}-\delta^{i k} G^{j},
$$

and satisfy the following commutation relations:

$$
\begin{array}{ll}
{\left[D, P^{i}\right]=+P^{i},} & {\left[D, G^{i}\right]=-G^{i},} \\
{\left[H, P^{i}\right]=0, \quad\left[C, P^{i}\right]=+G^{i},} & {\left[H, G^{i}\right]=-P^{i}, \quad\left[C, G^{i}\right]=0 .}
\end{array}
$$

Finally, we have the central extension with the "rest-mass" or the particle number,

$$
\left[P^{i}, G^{j}\right]=-\delta^{i j} M
$$

All the generators above are anti-Hermitian.

It is sometimes useful to introduce a Virasoro-like notation,

$$
L_{0} \equiv \frac{1}{2} D, \quad L_{-1} \equiv H, \quad L_{+1} \equiv C, \quad P_{-1 / 2}^{i} \equiv P^{i}, P_{+1 / 2}^{i} \equiv G^{i}, \quad M_{0} \equiv M .
$$

Then, the commutation relations can be compactly summarized as

$$
\left[L_{m}, L_{n}\right]=(m-n) L_{m+n}, \quad\left[L_{m}, P_{r}^{i}\right]=\left(\frac{1}{2} m-r\right) P_{m+r}^{i}, \quad\left[P_{r}^{i}, P_{s}^{j}\right]=(r-s) \delta^{i j} M_{r+s}
$$


Global frame. As explained in [25], the operator-state map naturally introduces the following recombination of generators:

$$
\begin{aligned}
& \widehat{L}_{0} \equiv \frac{1}{2}(-i H-i C), \quad \widehat{L}_{ \pm 1} \equiv \frac{1}{2}(-i H+i C \pm D), \\
& \widehat{P}_{ \pm 1 / 2}^{i}=\frac{1}{\sqrt{2}}\left(-i P^{i} \mp G^{i}\right), \quad \widehat{M}_{0}=-i M_{0} .
\end{aligned}
$$

The new generators also satisfy Virasoro-like commutation relations,

$$
\left[\widehat{L}_{m}, \widehat{L}_{n}\right]=(m-n) L_{m+n}, \quad\left[\widehat{L}_{m}, \widehat{P}_{r}^{i}\right]=\left(\frac{1}{2} m-r\right) \widehat{P}_{m+r}^{i},\left[\widehat{P}_{r}^{i}, \widehat{P}_{s}^{j}\right]=(r-s) \delta^{i j} \widehat{M}_{r+s},
$$

as well as the conjugation relations

$$
\left(\widehat{L}_{m}\right)^{\dagger}=L_{-m}, \quad\left(\widehat{P}_{r}^{i}\right)^{\dagger}=P_{-r}^{i}, \quad\left(\widehat{M}_{0}\right)^{\dagger}=\widehat{M}_{0} .
$$

Geometric realization In [1,2], a $(d+3)$-dimensional Schrödinger-invariant metric was presented. In our convention, the metric takes the form

$$
d s^{2}=-\frac{d t^{2}}{r^{4}}+\frac{2 d t d v+d \vec{x}^{2}+d r^{2}}{r^{2}} .
$$

The generators of the Schrödinger algebra are realized as Killing vectors of this metric,

$$
\begin{aligned}
& L_{m}=-t^{m+1} \partial_{t}-\frac{1}{2}(m+1) t^{m}\left(r \partial_{r}+x^{i} \partial_{i}\right)+\frac{1}{4} m(m+1) t^{m-1}\left(\vec{x}^{2}+r^{2}\right) \partial_{v}, \\
& P_{r}^{i}=t^{r+1 / 2} \partial^{i}-\left(r+\frac{1}{2}\right) t^{r-1 / 2} x^{i} \partial_{v}, \quad M_{m}=t^{m} \partial_{v}, \quad M_{i j}=x_{i} \partial_{j}-x_{j} \partial_{i} .
\end{aligned}
$$

A global coordinate for the geometry (2.16) was found in [26]. It is related to the Poincaré coordinate by the following transformation,

$$
t=\tan T, \quad r=\frac{R}{\cos T}, \quad \vec{x}=\frac{\vec{X}}{\cos T}, \quad v=V-\frac{1}{2}\left(R^{2}+\vec{X}^{2}\right) \tan T .
$$

In the new coordinate, the metric reads

$$
d s^{2}=-\frac{d T^{2}}{R^{4}}+\frac{2 d T d V-\left(\vec{X}^{2}+R^{2}\right) d T^{2}+d \vec{X}^{2}+d R^{2}}{R^{2}} .
$$

The global form of the Schrödinger generators get simplified in this coordinate,

$$
\begin{aligned}
& \widehat{L}_{0}=\frac{1}{2}\left(i \partial_{T}\right), \quad \widehat{L}_{ \pm 1}=\frac{1}{2} e^{ \pm 2 i T}\left[i \partial_{T}+i\left(\vec{X}^{2}+R^{2}\right) \partial_{V} \mp\left(X^{i} \partial_{X^{i}}+R \partial_{R}\right)\right], \\
& \widehat{P}_{ \pm 1 / 2}^{i}=\frac{1}{\sqrt{2}} e^{ \pm i T}\left(-i \partial_{X^{i}} \mp X^{i} \partial_{V}\right), \quad \widehat{M}_{0}=-i \partial_{V} .
\end{aligned}
$$

Schrödinger algebra in $d=2$ Let $J \equiv-i M^{12}$ be the $S O(2)$ rotation generator. It is useful to combine other generators according to their helicity ( $J$-eigenvalue) defined by

$$
[J, \mathcal{O}]=j \mathcal{O} .
$$

For example, $P_{r} \equiv P_{r}^{1}+i P_{r}^{2}$ has $j=+1$ and $\bar{P}_{r} \equiv P_{r}^{1}-i P_{r}^{2}$ has $j=-1$. In the helicity basis, the bosonic algebra can be rewritten as

$$
\left[L_{m}, L_{n}\right]=(m-n) L_{m+n}, \quad\left[L_{m}, P_{r}\right]=\left(\frac{1}{2} m-r\right) P_{m+r}, \quad\left[P_{r}, \bar{P}_{s}\right]=2(r-s) M_{r+s} .
$$

In what follows, we will denote operators with non-negative $j$ by unbarred operators $\mathcal{O}$ and their hermitian conjugates by barred operators $\overline{\mathcal{O}}$. 


\section{Super-Schrödinger algebra in $d=2$}

$\mathcal{N}=2$ super-Sch algebra This algebra was first introduced in [12] in the context of Chern-Simons systems. The notation $\mathcal{N}=2$ refers to the supersymmetry of the relativistic parent theory. In the "Poincaré frame", it has kinematical $(q, \bar{q})$, dynamical $(Q, \bar{Q})$ and conformal $(S, \bar{S})$ supercharges, and a $U(1) R$-symmetry.

Let us jump directly to the Virasoro-like notation in which the commutation relations take the simplest form. The supercharges are denoted by $q, Q_{-1 / 2} \equiv Q, Q_{+1 / 2} \equiv S$ and their conjugates. They transform under the $S O(2,1) \times U(1)_{J} \times U(1)_{R}$ subalgebra as

$$
\left[L_{m}, Q_{r}\right]=\left(\frac{1}{2} m-r\right) Q_{r}, \quad\left[L_{m}, q\right]=0,
$$

and

$$
\left[J, Q_{r}\right]=+\frac{1}{2} Q_{r}, \quad\left[R, Q_{r}\right]=+Q_{r}, \quad[J, q]=+\frac{1}{2} q, \quad[R, q]=-q .
$$

Their commutators with $P_{r}$ give

$$
\left[\bar{P}_{r}, Q_{s}\right]=(r-s) \bar{q}, \quad\left[\bar{P}_{r}, q\right]=0 .
$$

Finally, the anti-commutators among supercharges give

$$
\left\{\bar{Q}_{r}, Q_{s}\right\}=L_{r+s}+\frac{1}{2}(r-s)\left(J-\frac{3}{2} R\right), \quad\left\{q, Q_{r}\right\}=P_{r}, \quad\{\bar{q}, q\}=2 M .
$$

Note that $\left(L_{m}, Q_{r}, J-\frac{3}{2} R\right)$ form a closed sub-algebra, called $\operatorname{OS} p(2 \mid 1)$, isomorphic to the usual $\mathcal{N}=2$ superconformal algebra in a chiral sector of RNS superstring world-sheet.

$\mathcal{N}=6$ super-Sch algebra The ABJM theory has an $S U(4) R$-symmetry. The mass deformation breaks it into $S U(2)_{1} \times S U(2)_{2} \times U(1)_{R}$. The six supercharges participating in the $\mathcal{N}=2$ subalgebra are singlets of $S U(2)_{1} \times S U(2)_{2}$. The additional eight supercharges, which we call spectator supercharges, satisfy the following relations:

$$
\begin{aligned}
& {\left[L_{m}, q_{a \dot{a}}\right]=0, \quad\left[P_{r}, q_{a \dot{a}}\right]=0=\left[\bar{P}_{r}, q_{a \dot{a}}\right],} \\
& \left\{Q_{r}, q_{a \dot{a}}\right\}=0=\left\{\bar{Q}_{r}, q_{a \dot{a}}\right\}, \quad\left\{q, q_{a \dot{a}}\right\}=0=\left\{\bar{q}, q_{a \dot{a}}\right\}, \\
& {\left[J, q_{a \dot{a}}\right]=+\frac{1}{2} q_{a \dot{a}}, \quad\left[R, q_{a \dot{a}}\right]=0,} \\
& {\left[R^{a}, q_{c \dot{c}}\right]=-\delta_{\gamma}^{\alpha} q_{b \dot{c}}+\frac{1}{2} \delta_{b}^{a} q_{c \dot{c}}, \quad\left[R_{\dot{b}}^{\dot{a}}{ } q_{c \dot{c}}\right]=-\delta_{c}^{a} q_{\dot{c} \dot{b}}+\frac{1}{2} \delta_{\dot{b}}^{\dot{a}} q_{c \dot{c}},} \\
& \left\{\bar{q}^{a \dot{a}}, q_{b \dot{b}}\right\}=\frac{1}{2} \delta_{b}^{a} \delta_{\dot{b}}^{\dot{a}} M-\delta_{b}^{a} R_{\dot{b}}^{\dot{a}}+\delta_{\dot{b}}^{\dot{a}} R_{b}^{a},
\end{aligned}
$$

where $R_{b}^{a}, R_{b}^{\dot{a}}$ are the $S U(2)$ generators defined by

$$
\left[R_{b}^{a}, R_{d}^{c}\right]=\delta_{b}^{c} R_{d}^{a}-\delta_{d}^{a} R_{b}^{c}, \quad\left(R_{b}^{a}\right)^{\dagger}=R_{a}^{b} .
$$

The $\mathcal{N}=2$ subalgebra (2.26) still holds, except that the generator $R$ is replaced by $\tilde{R}$. In the field theory, the shift is partly due to an additional conserved quantity, namely, the fermion number $\Sigma$. The shifted $R$-charge is related to the original one by $\tilde{R}=(4 / 3) R-$ $(2 / 3) \Sigma$. From the commutation relations, we see that the shift is needed to make $q_{a \dot{a}}$ neutral under $J-\frac{3}{2} \tilde{R}$, which should hold because $q_{a \dot{a}}$ commutes with $Q_{r}$. It is not clear how to realize $\Sigma$ geometrically. 


\begin{tabular}{c|c|c|c|c} 
& $L_{0}$ & $J$ & $R$ & $\tilde{R}$ \\
\hline$Q$ & +1 & $+1 / 2$ & +1 & +1 \\
$S$ & -1 & $+1 / 2$ & +1 & +1 \\
$q$ & 0 & $+1 / 2$ & -1 & -1 \\
$q_{\alpha \dot{\alpha}}$ & 0 & $+1 / 2$ & 0 & $1 / 3$
\end{tabular}

Table 1: $U(1)$ quantum numbers of supercharges.

\subsection{Ansatz}

Recall the sequence of the $R$-symmetry breaking,

$$
S O(8) \supset U(1)_{B} \times S U(4) \supset U(1)_{B} \times S U(2)_{1} \times S U(2)_{2} \times U(1)_{R} .
$$

To see how these $R$-symmetries are realized geometrically, consider $S^{7}$ as a warped product of two $S^{3}$ s, and write down the metric as

$$
d s_{S^{7}}^{2}=d \alpha^{2}+\cos ^{2} \alpha d \Omega_{1}^{2}+\sin ^{2} \alpha d \Omega_{2}^{2} .
$$

We use the standard Euler-angle coordinates $(\theta, \phi, \psi)$ for each $S^{3}$ :

$$
d \Omega_{i}^{2}=\frac{1}{4}\left[d \theta_{i}^{2}+\sin ^{2} \theta_{i} d \phi_{i}^{2}+\left(d \psi_{i}-\cos \theta_{i} d \phi_{i}\right)^{2}\right] \quad(i=1,2, \text { no sum }) .
$$

We choose the orientations of the 3-spheres such that the $U(1)_{R}$ acts diagonally on $\psi_{1,2}$ and the $U(1)_{B}$ acts with an opposite relative sign.

Now, let us begin with $\mathrm{AdS}_{4} \times S^{7} / \mathbb{Z}_{k}$ and imagine taking the mass deformation and then the non-relativistic limit. The procedure will change the metric significantly, but the $R$-symmetries (2.29) as well as the time and space translation (in Poincaré patch) should be preserved throughout. Moreover, the fibration structure of the $U(1)_{B}$ and $U(1)_{R}$ angles over the two $S^{2}$ 's should be maintained.

In what follows, we will use the following notations

$$
\begin{aligned}
& w=\frac{1}{2}\left(\psi_{1}+\psi_{2}\right), \quad v=\frac{1}{2}\left(\psi_{1}-\psi_{2}\right), \\
& D w=d w-\frac{1}{2}\left(\cos \theta_{1} d \phi_{1}+\cos \theta_{2} \phi_{2}\right), \\
& D v=d v-\frac{1}{2}\left(\cos \theta_{1} d \phi_{1}-\cos \theta_{2} \phi_{2}\right), \\
& d \omega_{i}^{2}=\frac{1}{4}\left(d \theta_{i}^{2}+\sin ^{2} \theta_{i} d \phi_{i}^{2}\right),
\end{aligned}
$$

Metric We can try to write down the most general ansatz for the metric and the 4-form flux consistent with the Schrödinger symmetry, global symmetries as well as the fibration structure. Building upon the Schrödinger-invariant metric of [1,2],

$$
d s^{2}=-\frac{d t^{2}}{r^{4}}+\frac{2 d t d \psi+d r^{2}+d \vec{x}^{2}}{r^{2}}
$$


we propose our ansatz for the metric,

$$
\begin{aligned}
d s^{2}= & e^{2 c_{1}}\left(-c_{2} \frac{d t^{2}}{r^{4}}+\frac{2 d t\left(D v+c_{3} D w\right)+d r^{2}+d \vec{x}^{2}}{r^{2}}+\frac{4}{9} e^{2 h_{2}}(D w)^{2}\right) \\
& +e^{-4 c_{1}}\left(e^{-2 h_{2}} d y^{2}+\frac{4}{3} e^{2 h_{1}}\left(e^{+2 h_{3}} d \omega_{1}^{2}+e^{-2 h_{3}} d \omega_{2}^{2}\right)\right) .
\end{aligned}
$$

All the functions $\left(c_{1,2,3}, h_{0,1,2,3}\right)$ depend only on $y$, which is the only coordinate not constrained by the continuous symmetries of the geometry. We "gauge-fixed" the reparametriztion invariance in $y$ by a particular choice of $g_{y y}$. The numerical factors $4 / 9$ and $4 / 3$ are inserted for later convenience. The Schrödinger symmetry and $R$-symmetry allow for two more terms in the metric,

$$
r^{-2} d t d y, \quad D w d y
$$

but both of them can be removed by shifting $v$ and $w$ by $y$-dependent functions.

Orthonormal frame The metric ansatz (2.37) admits a natural orthonormal frame,

$$
\begin{aligned}
& e^{+}=\frac{e^{2 c_{1}}}{r^{2}} d t, \quad e^{-}=-\frac{c_{2}}{2 r^{2}} d t+D v+c_{3} D w, \\
& e^{1}=\frac{e^{c_{1}}}{r} d x^{1}, \quad e^{2}=\frac{e^{c_{1}}}{r} d x^{2}, \quad e^{7}=\frac{2}{3} e^{c_{1}+h_{2}} D w, \quad e^{8}=\frac{e^{c_{1}}}{r} d r, \quad e^{9}=e^{-2 c_{1}-h_{2}} d y, \\
& \left(e^{3}, e^{4} ; e^{5}, e^{6}\right)=\frac{1}{\sqrt{3}} e^{-2 c_{1}+h_{1}}\left(e^{+h_{3}}\left(\sigma_{1}, \sigma_{2}\right) ; e^{-h_{3}}\left(\tau_{1}, \tau_{2}\right)\right) .
\end{aligned}
$$

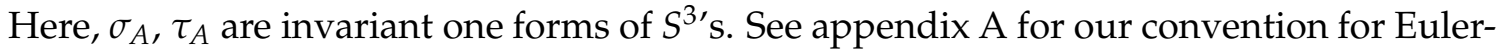
angle coordinates.

Flux To write down the general ansatz for the 4-form flux, we first collect all Schrödinger invariant $p$-forms in the "external" part of the metric:

$$
\left\{e^{+128}, e^{+12}, e^{+8}, e^{+}\right\}
$$

Note that all the invariant $p$-forms contain $e^{+}$. Combining these with invariant $p$-forms from the internal part, we arrive at the ansatz with ten unknown functions,

$$
\begin{aligned}
F= & e^{-3 c_{1}} e^{+8}\left[e^{-2 c_{1}} k_{1} e^{12}+e^{4 c_{1}-2 h_{1}}\left(e^{-2 h_{3}} k_{4,1} e^{34}+e^{+2 h_{3}} k_{4,2} e^{56}\right)\right] \\
& +e^{h_{2}} e^{+9}\left[e^{-2 c_{1}} k_{2} e^{12}+e^{4 c_{1}-2 h_{1}}\left(e^{-2 h_{3}} k_{5,1} e^{34}+e^{+2 h_{3}} k_{5,2} e^{56}\right)\right] \\
& +e^{c_{1}} e^{97}\left[e^{-3 c_{1}} k_{3} e^{+8}+e^{4 c_{1}-2 h_{1}}\left(e^{-2 h_{3}} k_{6,1} e^{34}+e^{+2 h_{3}} k_{6,2} e^{56}\right)\right] \\
& +e^{8 c_{1}-4 h_{1}} k_{7} e^{3456} .
\end{aligned}
$$

Here, we are using the shorthand notation $e^{a b}=e^{a} \wedge e^{b}$, etc. and assuming wedge products among differential forms. We inserted compensating factors of metric coefficients so 
that the Bianchi identity $(d F=0)$ maintains the simple form,

$$
\begin{aligned}
k_{1}^{\prime}+4 k_{2} & =0, \\
k_{4,1}^{\prime}+2 k_{5,1}-k_{3} & =0, \\
k_{4,2}^{\prime}+2 k_{5,2}-k_{3} & =0, \\
k_{7}^{\prime}-\left(k_{6,1}+k_{6,2}\right) & =0 .
\end{aligned}
$$

There are three more terms allowed by the symmetries, $\left\{e^{+127}, e^{+347}, e^{+567}\right\}$, but they are excluded by the Bianchi identity.

Parity symmetry There is a discrete $\mathbb{Z}_{2}$ symmetry exchanging the two 2-spheres which acts as a parity $y \rightarrow-y$. The unknown functions have the following parity eigenvalues,

$$
\begin{array}{r}
\text { Even : } c_{1}, c_{2}, h_{1}, h_{2}, k_{1},\left(k_{4,1}+k_{4,2}\right),\left(k_{5,1}-k_{5,2}\right),\left(k_{6,1}+k_{6,2}\right) . \\
\text { Odd : } c_{3}, h_{3}, k_{2}, k_{3},\left(k_{4,1}-k_{4,2}\right),\left(k_{5,1}+k_{5,2}\right),\left(k_{6,1}-k_{6,2}\right), k_{7} .
\end{array}
$$

\section{Solution and a Sketch of the Computation}

Having written out the most general ansatz, in this section we give a quick overview of our methods, summarize the equations imposed on the unknown functions in the ansatz, write down the solution and discuss its properties. The details of the computation will be postponed until the next section.

\subsection{Methods}

Supersymmetric solutions of M-theory satisfy the Killing spinor equation,

$$
\delta_{\epsilon} \psi_{M}=\nabla_{M} \epsilon+\frac{1}{12 \cdot 4 !} F_{I J K L}\left(F_{M}^{I J K L}-8 \delta_{M}^{I} \Gamma^{J K L}\right) \epsilon=0 .
$$

See appendix A for our conventions for 11-dimensional supergravity. Our approach to the problem will hinge upon two standard tools used for finding supersymmetric solutions, namely, the spinorial Lie derivative and the G-structure.

To begin with, the Lie derivative of a spinor $\epsilon$ with respect to a Killing vector $K$ may be defined as in [27]

$$
\mathfrak{L}_{K} \epsilon=K^{m} \nabla_{m} \epsilon+\frac{1}{4}\left(\nabla_{a} K_{b}\right) \Gamma^{a b} \epsilon .
$$

In general, the spinorial Lie derivative gives a geometric realization of the algebra,

$$
\left[K, Q_{1}\right]=Q_{2} \Longleftrightarrow \mathfrak{L}_{K} \epsilon_{Q_{1}}=\epsilon_{Q_{2}} .
$$

From the metric ansatz (2.37), one may then write out the spinoral Lie derivatives associated to the various Killing directions. The Lie derivatives of the spinors, via the super Schrödinger algebra discussed in section 2, determine all coordinate dependence other 
than the $y$-direction of the two dynamical supercharges $Q$. Once $Q$ are determined, the kinematical $q$ and conformal $S$ supercharges also may be worked out from the algebra.

Adopting the language of G-structures to M-theory was initiated in [28,29]. Assuming the existence of Killing spinors $\left\{\epsilon_{i}\right\}$, one constructs the following differential forms

$$
\begin{aligned}
K_{i j} & =\left(\bar{\epsilon}_{i} \Gamma_{a} \epsilon_{j}\right) e^{a}, \\
\Omega_{i j} & =\frac{1}{2}\left(\bar{\epsilon}_{i} \Gamma_{a b} \epsilon_{j}\right) e^{a b}, \\
\Sigma_{i j} & =\frac{1}{5 !}\left(\bar{\epsilon}_{i} \Gamma_{a b c d e} \epsilon_{j}\right) e^{a b c d e} .
\end{aligned}
$$

The Killing spinor equations imply that $K_{i j}$ are Killing vectors, so that (3.4) becomes a geometric representation of the algebra

$$
\left\{Q_{i}, Q_{j}\right\}=K_{i j}
$$

In addition, the KSE give a set of algebraic and differential relations among $(K, \Omega, \Sigma)$. These relations are equivalent to the original KSE by construction, but are often easier to solve and illuminate the geometric structure more clearly. For the purpose of this paper, however, it turns out to be more straightforward to analyze the KSE directly, while keeping in mind the lessons from [28,29].

We will demand that our ansatz admit the six supercharges of $\mathcal{N}=2$ super-Sch algebra. The kinematical supercharges $(q, \bar{q})$ correspond to null Killing spinors studied in [29], whereas the dynamical supercharges $(Q, \bar{Q})$ correspond to time-like Killing spinors studied in [28]. To use the results of [29] directly, we first focus on the real combination $\epsilon=\frac{1}{2}(q+\bar{q})$ which satisfies the two projection conditions

$$
\left.\Gamma^{3456} \epsilon=-\epsilon \quad \text { singlet under } S U(2)_{1} \times S U(2)_{2}\right), \quad \Gamma^{+} \epsilon=0,
$$

and defines an $S U(7)$ structure explained in [29]. Restoring both components $(q, \bar{q})$ then defines an SU(4) sub-structure of the Spin(7) structure. Having started by introducing an ansatz, making the G-structure manifest entails a small frame rotation from the original frame to the canonical G-structure frame. Similarly, for $(Q, \bar{Q})$ we find an $S U(4)$ substructure of the $S U(5)$ structure introduced in [28]. The conformal supercharges $(S, \bar{S})$ do not yield any new information because they are related to $(Q, \bar{Q})$ by the conformal symmetry generator and all bosonic symmetries are already built into our ansatz.

\subsection{Killing spinor equations: summary}

After a somewhat lengthy analysis to be presented in section 4 , the Killing spinor equations for the six supercharges give rise to a number of coupled equations for all the unknown variables. They may be divided into three blocks. 
1. Block $\mathrm{A}$ : The equations for $\left(c_{1}, h_{1}, h_{2}, h_{3}\right)$ decouple from all other variables.

$$
\begin{aligned}
& 4 h_{1}^{\prime}-h_{2}^{\prime}=-c_{1}^{\prime}\left(2 h_{1}^{\prime}+h_{2}^{\prime}\right)^{2} e^{6 c_{1}+2 h_{2},} \\
& 9 c_{1}^{\prime}=\left(9 c_{1}^{\prime}-4 h_{1}^{\prime}+h_{2}^{\prime}\right) e^{2 h_{2},}, \\
& 2 h_{1}^{\prime}+h_{2}^{\prime}=6\left(h_{1}^{\prime}+h_{3}^{\prime}\right) e^{-6 c_{1}+2 h_{1}-2 h_{2}+2 h_{3},} \\
& h_{3}^{\prime} \cosh \left(2 h_{3}\right)=-h_{1}^{\prime} \sinh \left(2 h_{3}\right) .
\end{aligned}
$$

The following auxiliary equations will also be useful,

$$
\begin{aligned}
& \cos \zeta=e^{h_{2}}, \\
& \sin \zeta=-\frac{1}{3}\left(2 h_{1}^{\prime}+h_{2}^{\prime}\right) e^{3 c_{1}+2 h_{2}}=\frac{1}{3 c_{1}^{\prime}}\left(-\zeta^{\prime} \cos \zeta+2 e^{-3 c_{1}}\right) .
\end{aligned}
$$

2. Block B : With the solutions of Block $A$ as an input, we can solve the equations for $\left(c_{3}, k_{1}, k_{2}, k_{3}\right)$.

$$
\begin{aligned}
& k_{2}=-k_{3}, \\
& k_{1}=-\frac{6 c_{3}}{\sin \zeta} e^{3 c_{1}} \\
& 3 c_{3}^{\prime}+k_{1} e^{-6 c_{1}}=6 \sin \zeta\left(c_{3} \cosh \left(2 h_{3}\right)-\sinh \left(2 h_{3}\right)\right) e^{3 c_{1}-2 h_{1}} \\
& 3 c_{3}^{\prime}=2\left(k_{1} e^{-6 c_{1}} \frac{h_{1}^{\prime}-h_{2}^{\prime}}{2 h_{1}^{\prime}+h_{2}^{\prime}}-k_{3} e^{-3 c_{1}} \sin \zeta\right) .
\end{aligned}
$$

3. Block $\mathrm{C}$ : The last metric component $c_{2}$ and all the remaining flux components are determined algebraically by the solutions of Block A and Block B.

$$
\begin{aligned}
& c_{2}=\left(\frac{1}{4} k_{1} e^{-3 c_{1}}\right)^{2}, \\
& k_{4,1}=-\frac{3}{2}\left(c_{3}+1\right) e^{3 c_{1}} \sin \zeta-\frac{1}{4} k_{1}\left(2 e^{-6 c_{1}+2 h_{1}+2 h_{3}}-e^{2 h_{2}}\right), \\
& k_{4,2}=-\frac{3}{2}\left(c_{3}-1\right) e^{3 c_{1}} \sin \zeta-\frac{1}{4} k_{1}\left(2 e^{-6 c_{1}+2 h_{1}-2 h_{3}}-e^{2 h_{2}}\right), \\
& k_{5,1}=-\frac{3}{2}\left(c_{3}-1\right) e^{+4 h_{2}} \\
& k_{5,2}=-\frac{3}{2}\left(c_{3}+1\right) e^{-4 h_{2}} \\
& k_{6,1}=-\frac{h_{1}^{\prime}+2 h_{2}^{\prime}+3 h_{3}^{\prime}}{3\left(h_{1}^{\prime}+h_{3}^{\prime}\right)} e^{2 h_{2}} \\
& k_{6,2}=-\frac{h_{1}^{\prime}+2 h_{2}^{\prime}-3 h_{3}^{\prime}}{3\left(h_{1}^{\prime}-h_{3}^{\prime}\right)} e^{2 h_{2}} \\
& k_{7}=6 c_{1}^{\prime} e^{-6 c_{1}+4 h_{1}}
\end{aligned}
$$

\subsection{Solution}

Rather remarkably, the set of coupled equations listed above can be solved completely in a closed form. We first note that (3.10) can be readily integrated to give

$$
\left|\sinh \left(2 h_{3}\right)\right|=\beta e^{-2 h_{1}},
$$


where $\beta$ is an integration constant. But, for any non-vanishing $\beta$, the metric is singular at $h_{3}=0$. To avoid the singularity, we are forced to set $\beta=0$. Then, $h_{3}$ vanishes identically.

Integrating the second half of (3.12), we find

$$
\partial_{y}\left(\sin \zeta e^{3 c_{1}}\right)=2 \Rightarrow \sin \zeta e^{3 c_{1}}=2 y .
$$

In principle, another integration constant should be introduced here. But, a non-zero constant turns out to induce terms proportional to $(\log y)$ in $e^{2 h_{2}}$ and $e^{6 c_{1}}$, leading to a singular metric. So, we drop the constant.

Inserting the first half of (3.12) to the LHS of (3.10) and integrating, we find

$$
e^{2 h_{1}}=p-y^{2},
$$

Multiplying each side of (3.7) and (3.9), eliminating $c_{1}^{\prime}$ by using (3.8) and integrating, we obtain the solution for $h_{2}$,

$$
e^{2 h_{2}}=\frac{-3 y^{4}-2 c y^{3}-6 p y^{2}+p^{2}}{\left(p-y^{2}\right)^{2}},
$$

Finally, solving $(3.9)$ for $e^{6 c_{1}}$, we find

$$
e^{6 c_{1}}=\frac{\left(p-y^{2}\right)^{2}}{p+\frac{1}{2} c y+y^{2}} .
$$

Here, $c$ an $p$ are integral constants. By a rescaling of $y$ and $c$, as well as an overall rescaling of the whole metric, we can always set $p=1$. Written in this form, the metric components we have found are essentially identical to those of [15]. The condition for global regularity of the solution discussed in [15] can be carried over to our case; we require the following constraints on $c$ and $y$

$$
0 \leq c<4, \quad y_{1} \leq y \leq y_{2} ，
$$

where $y_{1}$ and $y_{2}$ are the two real roots of the equation $e^{2 h_{2}}=\cos ^{2} \zeta=0$. In addition, we must choose the period of $w$ to be $2 \pi$ to have a smooth geometry at both $y_{1}$ and $y_{2}$. The regular solutions with these conditions are $S^{2}$ fibrations over $S^{2} \times S^{2}$ [15].

It is straightforward to solve equations in Block B. For instance, by combining the second and third equations, we obtain

$$
c_{3}=\frac{4 b y}{3 y\left(1-y^{2}\right)} .
$$

with $b$ being an integration constant. Other equations in Block B are easier to solve. Block $\mathrm{C}$ equations then determine the remaining unknown functions algebraically.

In summary, we have obtained the most general solution compatible with $\mathcal{N}=2$ super-Schrödinger symmetry and the global symmetry discussed in section 2 . The solution is parameterized by two real constants $b$ and $c$. The final form of the solution may be most neatly captured in terms of two quadratic polynomials,

$$
g_{1}=1-y^{2}, \quad g_{2}=1+\frac{1}{2} c y+y^{2} .
$$


The metric components are

$$
\begin{aligned}
& e^{6 c_{1}}=g_{1}^{2} g_{2}^{-1}, \quad c_{2}=b^{2} g_{1}^{-2} g_{2}^{-1}, \quad c_{3}=\frac{4}{3} b y g_{1}^{-2}, \\
& e^{2 h_{1}}=g_{1}, \quad e^{2 h_{2}}=1-4 y^{2} e^{-6 c_{1}}, \quad e^{2 h_{3}}=1 ，
\end{aligned}
$$

and the flux components are

$$
\begin{array}{ll}
k_{1}=-4 b g_{2}^{-1}, \quad k_{2}=-b g_{2}^{\prime} g_{2}^{-2}, \quad k_{3}=b g_{2}^{\prime} g_{2}^{-2}, \\
k_{4,1}=-3 y+b\left(2 g_{1}^{-1}-g_{2}^{-1}\right) & k_{4,2}=+3 y+b\left(2 g_{1}^{-1}-g_{2}^{-1}\right), \\
k_{5,1}=+\frac{3}{2}-2 y b g_{1}^{-2} & k_{5,2}=-\frac{3}{2}-2 y b g_{1}^{-2}, \\
k_{6,1}=k_{6,2}=1-4 g_{2} g_{1}^{-2}, & k_{7}=-4 g_{2}^{\prime} g_{1}^{-1}+2 g_{1}^{\prime}+3 g_{2}^{\prime} .
\end{array}
$$

Ooguri-Park solution In hindsight, our solution can be regarded as a one-parameter generalization of the recently discovered solution of Ooguri and Park [14]. Their solution was obtained by a judicious deformation of a known $A d S_{5}$ solution in M-theory [15]. It has two parameters $\beta$ and $c$. It preserves two supercharges for $\beta \neq 0$ and six supercharges for $\beta=0$.

It is easy to compare our solution with the Ooguri-Park solution. To be consistent with normalization conventions, we should relate our coordinates to theirs by

$$
t=-2 x^{+}, \quad v=\frac{1}{2} x^{-}, \quad w=\frac{1}{2} \psi,
$$

and set $n=1$ in their solution, although it is easy to generalize the comparison for arbitrary $n$. Then it is immediately clear that our solution with $b=0$ is identical to their solution with $\beta=0$. Note that the norm of the 'time' Killing vector $\partial_{t}$ vanishes when $b=0$. In this sense, the $b \rightarrow 0$ limit is not smooth;

Non-existence of spectator supercharges Our original goal was to find the gravity dual of the NR-ABJM theory with 14 supercharges. But, the Killing spinor equations for the $\operatorname{six} \mathcal{N}=2$ supercharges have already determined all unknown functions in our ansatz completely. Proceeding with the same methods, it is not difficult to show that our solution does not admit the other eight 'spectator' supercharges. We leave the technical proof of this 'no-go' theorem and discussion of physical implications for the concluding section.

\section{Details of the Computation}

In this section, we present a detailed analysis of Killing spinor equations using the methods mentioned in subsection 3.1, which yielded the set of equations summarized in subsection 3.2 .

\subsection{Killing spinor equations}

We want to solve the Killing spinor equation,

$$
\delta \psi_{m}=\nabla_{m} \epsilon+\frac{1}{12}\left(\Gamma_{m} \mathbf{F}-3 \mathbf{F}_{m}\right) \epsilon=0 \quad\left(\nabla_{m} \epsilon \equiv \partial_{m} \epsilon+\frac{1}{4}\left(\omega_{m}\right)_{a b} \Gamma^{a b} \epsilon\right) .
$$


where we defined, following [14],

$$
\mathbf{F} \equiv \frac{1}{4 !} F_{m n p q} \Gamma^{m n p q}, \quad \mathbf{F}_{m} \equiv \frac{1}{2}\left[\Gamma_{m}, \mathbf{F}\right]
$$

Our ansatz for the flux (2.41) obviously gives

$$
\begin{aligned}
\mathbf{F}= & e^{-3 c_{1}} \Gamma^{+8}\left(e^{-2 c_{1}} k_{1} \Gamma^{12}+e^{4 c_{1}-2 h_{1}}\left(e^{-2 h_{3}} k_{4,1} \Gamma^{34}+e^{+2 h_{3}} k_{4,2} \Gamma^{56}\right)\right) \\
& +e^{h_{2}} \Gamma^{+9}\left(e^{-2 c_{1}} k_{2} \Gamma^{12}+e^{4 c_{1}-2 h_{1}}\left(e^{-2 h_{3}} k_{5,1} \Gamma^{34}+e^{+2 h_{3}} k_{5,2} \Gamma^{56}\right)\right) \\
& +e^{c_{1}} \Gamma^{97}\left(e^{-3 c_{1}} k_{3} \Gamma^{+8}+e^{4 c_{1}-2 h_{1}}\left(e^{-2 h_{3}} k_{6,1} \Gamma^{34}+e^{+2 h_{3}} k_{6,2} \Gamma^{56}\right)\right) \\
& +e^{8 c_{1}-4 h_{1}} k_{7} \Gamma^{3456},
\end{aligned}
$$

and (in the orthonormal basis)

$$
\begin{aligned}
\mathbf{F}_{+}= & e^{-3 c_{1}} \Gamma^{8}\left(e^{-2 c_{1}} k_{1} \Gamma^{12}+e^{4 c_{1}-2 h_{1}}\left(e^{-2 h_{3}} k_{4,1} \Gamma^{34}+e^{+2 h_{3}} k_{4,2} \Gamma^{56}\right)+e^{c_{1}} k_{3} \Gamma^{97}\right) \\
& +e^{h_{2}} \Gamma^{9}\left(e^{-2 c_{1}} k_{2} \Gamma^{12}+e^{4 c_{1}-2 h_{1}}\left(e^{-2 h_{3}} k_{5,1} \Gamma^{34}+e^{+2 h_{3}} k_{5,2} \Gamma^{56}\right)\right), \\
\mathbf{F}_{-}= & 0, \\
\mathbf{F}_{1}= & -\Gamma^{+2}\left(e^{-5 c_{1}} k_{1} \Gamma^{8}+e^{-2 c_{1}+h_{2}} k_{2} \Gamma^{9}\right), \\
\mathbf{F}_{2}= & +\Gamma^{+1}\left(e^{-5 c_{1}} k_{1} \Gamma^{8}+e^{-2 c_{1}+h_{2}} k_{2} \Gamma^{9}\right), \\
\mathbf{F}_{3}= & +\Gamma^{4}\left(e^{4 c_{1}-2 h_{1}-2 h_{3}}\left(e^{-3 c_{1}} k_{4,1} \Gamma^{+8}+e^{h_{2}} k_{5,1} \Gamma^{+9}+e^{c_{1}} k_{6,1} \Gamma^{97}\right)+e^{8 c_{1}-4 h_{1}} k_{7} \Gamma^{56}\right), \\
\mathbf{F}_{4}= & -\Gamma^{3}\left(e^{4 c_{1}-2 h_{1}-2 h_{3}}\left(e^{-3 c_{1}} k_{4,1} \Gamma^{+8}+e^{h_{2}} k_{5,1} \Gamma^{+9}+e^{c_{1}} k_{6,1} \Gamma^{97}\right)+e^{8 c_{1}-4 h_{1}} k_{7} \Gamma^{56}\right), \\
\mathbf{F}_{5}= & +\Gamma^{6}\left(e^{4 c_{1}-2 h_{1}+2 h_{3}}\left(e^{-3 c_{1}} k_{4,2} \Gamma^{+8}+e^{h_{2}} k_{5,2} \Gamma^{+9}+e^{c_{1}} k_{6,2} \Gamma^{97}\right)+e^{8 c_{1}-4 h_{1}} k_{7} \Gamma^{34}\right), \\
\mathbf{F}_{6}= & -\Gamma^{5}\left(e^{4 c_{1}-2 h_{1}+2 h_{3}}\left(e^{-3 c_{1}} k_{4,2} \Gamma^{+8}+e^{h_{2}} k_{5,2} \Gamma^{+9}+e^{c_{1}} k_{6,2} \Gamma^{97}\right)+e^{8 c_{1}-4 h_{1}} k_{7} \Gamma^{34}\right), \\
\mathbf{F}_{7}= & -e^{-2 c_{1}} k_{3} \Gamma^{+89}-e^{5 c_{1}-2 h_{1}} \Gamma^{9}\left(e^{-2 h_{3}} k_{6,1} \Gamma^{34}+e^{+2 h_{3}} k_{6,2} \Gamma^{56}\right), \\
\mathbf{F}_{8}= & -e^{-3 c_{1}} \Gamma^{+}\left(e^{-2 c_{1}} k_{1} \Gamma^{12}+e^{4 c_{1}-2 h_{1}}\left(e^{-2 h_{3}} k_{4,1} \Gamma^{34}+e^{+2 h_{3}} k_{4,2} \Gamma^{56}\right)+e^{c_{1}} k_{3} \Gamma^{97}\right), \\
\mathbf{F}_{9}= & -e^{h_{2}} \Gamma^{+}\left(e^{-2 c_{1}} k_{2} \Gamma^{12}+e^{4 c_{1}-2 h_{1}}\left(e^{-2 h_{3}} k_{5,1} \Gamma^{34}+e^{+2 h_{3}} k_{5,2} \Gamma^{56}\right)\right) \\
& +e^{c_{1}} \Gamma^{7}\left(e^{-3 c_{1}} k_{3} \Gamma^{+8}+e^{4 c_{1}-2 h_{1}}\left(e^{-2 h_{3}} k_{6,1} \Gamma^{34}+e^{+2 h_{3}} k_{6,2} \Gamma^{56}\right)\right) .
\end{aligned}
$$

We also list the contribution from the spin-connection, $\omega_{m} \equiv \frac{1}{2}\left(\omega_{m}\right)_{a b} \Gamma^{a b}$ (again in the 
orthonormal basis):

$$
\begin{aligned}
\omega_{+}= & -e^{-3 c_{1}} c_{2} \Gamma^{8+}+e^{-c_{1}} \Gamma^{8-}+\frac{1}{2} e^{h_{2}} c_{2}^{\prime} \Gamma^{9+}-e^{2 c_{1}+h_{2}} c_{1}^{\prime} \Gamma^{9-}-\frac{3}{4} e^{c_{1}} c_{3}^{\prime} \Gamma^{97} \\
& +\left\{\frac{1}{2} e^{-2 c_{1}} c_{2}-\frac{3}{4} e^{4 c_{1}-2 h_{1}-2 h_{3}}\left(1+c_{3}\right)\right\} \Gamma^{34}-\left\{\frac{1}{2} e^{-2 c_{1}} c_{2}-\frac{3}{4} e^{4 c_{1}-2 h_{1}+2 h_{3}}\left(1-c_{3}\right)\right\} \Gamma^{56}, \\
\omega_{-}= & \Gamma^{34}-\Gamma^{56}-e^{-c_{1}} \Gamma^{+8}+e^{2 c_{1}+h_{2}} c_{1}^{\prime} \Gamma^{+9}, \\
\omega_{1}= & -e^{-c_{1}} \Gamma^{18}+e^{2 c_{1}+h_{2}} c_{1}^{\prime} \Gamma^{19}, \\
\omega_{2}= & -e^{-c_{1}} \Gamma^{28}+e^{2 c_{1}+h_{2}} c_{1}^{\prime} \Gamma^{29}, \\
\omega_{3}= & +\frac{3}{4} e^{4 c_{1}-2 h_{1}-2 h_{3}}\left(1+c_{3}\right) \Gamma^{4+}+\frac{1}{2} e^{5 c_{1}-2 h_{1}+h_{2}-2 h_{3}} \Gamma^{47}+e^{2 c_{1}+h_{2}}\left(-2 c_{1}^{\prime}+h_{1}^{\prime}+h_{3}^{\prime}\right) \Gamma^{39}, \\
\omega_{4}= & -\frac{3}{4} e^{4 c_{1}-2 h_{1}-2 h_{3}}\left(1+c_{3}\right) \Gamma^{3+}-\frac{1}{2} e^{5 c_{1}-2 h_{1}+h_{2}-2 h_{3}} \Gamma^{37}+e^{2 c_{1}+h_{2}}\left(-2 c_{1}^{\prime}+h_{1}^{\prime}+h_{3}^{\prime}\right) \Gamma^{49}, \\
\omega_{5}= & -\frac{3}{4} e^{4 c_{1}-2 h_{1}+2 h_{3}}\left(1-c_{3}\right) \Gamma^{6+}+\frac{1}{2} e^{5 c_{1}-2 h_{1}+h_{2}+2 h_{3}} \Gamma^{67}+e^{2 c_{1}+h_{2}}\left(-2 c_{1}^{\prime}+h_{1}^{\prime}-h_{3}^{\prime}\right) \Gamma^{59}, \\
\omega_{6}= & +\frac{3}{4} e^{4 c_{1}-2 h_{1}+2 h_{3}}\left(1-c_{3}\right) \Gamma^{5+}-\frac{1}{2} e^{5 c_{1}-2 h_{1}+h_{2}+2 h_{3}} \Gamma^{57}+e^{2 c_{1}+h_{2}}\left(-2 c_{1}^{\prime}+h_{1}^{\prime}-h_{3}^{\prime}\right) \Gamma^{69}, \\
\omega_{7}= & \frac{1}{2}\left\{3 e^{-c_{1}-h_{2}}\left(1-c_{3}\right)-e^{5 c_{1}-2 h_{1}+h_{2}-2 h_{3}}\right\} \Gamma^{34} \\
& +\frac{1}{2}\left\{3 e^{-c_{1}-h_{2}}\left(1+c_{3}\right)-e^{5 c_{1}-2 h_{1}+h_{2}+2 h_{3}}\right\} \Gamma^{56} \\
& +\frac{3}{4} e^{c_{1}} c_{3}^{\prime} \Gamma^{+9}-e^{2 c_{1}+h_{2}}\left(c_{1}^{\prime}+h_{2}^{\prime}\right) \Gamma^{97}, \\
\omega_{8}= & -e^{-c_{1}}\left(1-\Gamma^{-} \Gamma^{+}\right)+e^{2 c_{1}+h_{2}} c_{1}^{\prime} \Gamma^{89}, \\
\omega_{9}= & e^{2 c_{1}+h_{2}} c_{1}^{\prime}\left(1-\Gamma^{-} \Gamma^{+}\right)+\frac{3}{4} e^{c_{1}} c_{3}^{\prime} \Gamma^{7+} .
\end{aligned}
$$

\subsection{Spinorial Lie derivatives}

The Lie derivative of a spinor with respect to a Killing vector is defined by

$$
\mathfrak{L}_{V} \epsilon=V^{m} \nabla_{m} \epsilon+\frac{1}{4}\left(\nabla_{a} V_{b}\right) \Gamma^{a b} \epsilon .
$$

For the Killing vectors under consideration, the Lie derivatives are given by

$$
\begin{aligned}
\mathfrak{L}_{H} \epsilon= & -\partial_{t} \epsilon, \\
\mathfrak{L}_{D} \epsilon= & \left(-2 t \partial_{t}-x^{i} \partial_{i}-r \partial_{r}\right) \epsilon, \\
\mathfrak{L}_{C} \epsilon= & {\left[-t^{2} \partial_{t}-t\left(x^{i} \partial_{i}+r \partial_{r}\right)-\frac{1}{2} e^{-c_{1}} r \Gamma^{+}\left(x_{i} \Gamma^{i}+r \Gamma^{8}\right)\right] \epsilon } \\
& +\frac{1}{2}\left(\vec{x}^{2}+r^{2}\right)\left[\partial_{v}+\frac{1}{2}\left(\Gamma^{34}-\Gamma^{56}\right)\right] \epsilon, \\
\mathfrak{L}_{P_{i}} \epsilon= & \partial_{i} \epsilon \\
\mathfrak{L}_{G_{i}} \epsilon= & {\left[t \partial_{i}+\frac{1}{2} e^{-c_{1}} r \Gamma^{+i}\right] \epsilon-x^{i}\left[\partial_{v}+\frac{1}{2}\left(\Gamma^{34}-\Gamma^{56}\right)\right] \epsilon, } \\
\mathfrak{L}_{J} \epsilon= & {\left[x_{1} \partial_{2}-x_{2} \partial_{1}+\frac{1}{2} \Gamma^{12}\right] \epsilon, } \\
\mathfrak{L}_{M} \epsilon= & {\left[\partial_{v}+\frac{1}{2}\left(\Gamma^{34}-\Gamma^{56}\right)\right] \epsilon, } \\
\mathfrak{L}_{R} \epsilon= & {\left[\partial_{w}+\frac{1}{2}\left(\Gamma^{34}+\Gamma^{56}\right)\right] \epsilon, } \\
\mathfrak{L}_{V_{A}} \epsilon= & V_{A} \epsilon, \\
\mathfrak{L}_{V_{A}^{\prime}} \epsilon= & V_{A}^{\prime} \epsilon .
\end{aligned}
$$


We expect $\mathfrak{L}_{M} \epsilon=0$ for all supercharges, which simplifies $\mathfrak{L}_{K} \epsilon$ and $\mathfrak{L}_{G_{i}} \epsilon$ somewhat. Here, $V_{A}$ and $V_{A}^{\prime}$ are Killing vectors for the $S U(2)_{1} \times S U(2)_{2}$ symmetry (see appendix A).

The dependence of Killing spinors on each coordinate is fixed by the Lie derivatives to a large extent. For dynamical supercharges $Q$, we find

$$
\mathfrak{L}_{H} \epsilon_{Q}=\mathfrak{L}_{P_{i}} \epsilon_{Q}=\mathfrak{L}_{V_{A}} \epsilon_{Q}=\mathfrak{L}_{V_{A}^{\prime}} \epsilon_{Q}=0, \mathfrak{L}_{D} \epsilon_{Q}=\epsilon_{Q} \Rightarrow \epsilon_{Q}=\frac{e^{c_{1}}}{r} \eta(y),
$$

The fact that $Q$ is singlet under $S U(2)_{1} \times S U(2)_{2}$ implies that $Q$ is independent of all 'three-sphere' coordinates $\left(v, w, \theta_{i}, \phi_{i}\right)$. Then, by $\mathfrak{L}_{M} \epsilon=0$, we find $\Gamma^{3456} \eta=-\eta$. Next, we can use $[G, \bar{Q}]=q$ to get

$$
\epsilon_{q}=\Gamma^{+}\left(\frac{\Gamma^{1}+i \Gamma^{2}}{2}\right) \eta^{c},
$$

where $\eta^{c}$ denotes the charge conjugation of $\eta$. Note that $\Gamma^{+} \epsilon_{q}=0$ holds automatically. Similarly, we can use $[K, Q]=S$ to get

$$
\epsilon_{S}=\left[\frac{t}{r} e^{c_{1}}-\frac{1}{2} \Gamma^{+}\left(x_{i} \Gamma^{i}+r \Gamma^{8}\right)\right] \eta .
$$

All six supercharges $(Q, S, q)$ of the $\mathcal{N}=2$ subalgebra are mapped to each other by the bosonic generators. As a consequence, they all share the same $\eta(y)$ and are independent of $\left(v, w, \theta_{i}, \phi_{i}\right)$ coordinates.

\subsection{Kinematical supercharges and null Killing spinor}

G-structure In our problem, we have a pair of Killing spinors corresponding to a null Killing vector; recall from (2.26) that $\{\bar{q}, q\}=2 M$. The fully general analysis of the geometry with a single (real) null Killing spinor was done in [29]. To import their language, we focus on the real combination $\epsilon=\frac{1}{2}\left(\epsilon_{q}+\epsilon_{\bar{q}}\right)$ for the moment.

The algebraic relations of [29] on a null Killing spinor can be summarized as follows. They take the orthonormal frame

$$
d s^{2}=2 e^{+} e^{-}+e^{i} e^{i}+e^{9} e^{9},
$$

with $i=1, \cdots, 8$ and

$$
K=e^{+}
$$

They further choose the Killing spinor to satisfy

$$
\Gamma_{1234} \epsilon=\Gamma_{3456} \epsilon=\Gamma_{5678} \epsilon=\Gamma_{1357} \epsilon=-\epsilon, \quad \Gamma^{+} \epsilon=0 .
$$

These conditions automatically implies $\Gamma^{9} \epsilon=\epsilon$. Next, they show that this spinor defines a $\operatorname{Spin}(7)$ structure within the $\mathbb{R}^{8}$ subspace of the tangent bundle. In particular, they find

$$
\Omega=e^{+} \wedge e^{9}, \quad \Sigma=e^{+} \wedge \Phi,
$$


where $\Phi$ is the invariant four form defining the embedding of Spin(7) into Spin(8),

$$
\begin{aligned}
-\Phi= & e^{1234}+e^{1256}+e^{1278}+e^{3456}+e^{3478}+e^{5678} \\
& +e^{1357}-e^{1368}-e^{1458}-e^{1467}-e^{2358}-e^{2367}-e^{2457}+e^{2468} .
\end{aligned}
$$

Our choice of the orthonormal frame (2.39) is such that $e^{+}$is the dual one-form of $M$ in agreement with [29]. We also showed already that parts of the conditions (4.13), namely, $\Gamma^{3456} \epsilon=-\epsilon$ and $\Gamma^{+} \epsilon=0$ hold for the kinematical supercharges. On the other hand, it is not clear whether the $(8+1)$ splitting in the canonical G-structure frame (4.11) agrees with our original choice of the frame (2.39). In fact, we will see that the two frames are related to each other by a $y$-dependent rotation on the (89)-plane.

Killing spinor equations We showed earlier that the kinematical supercharges satisfy

$$
\left.\Gamma^{+} \epsilon=0, \quad \Gamma^{3456} \epsilon=-\epsilon, \quad \partial_{m} \epsilon=0 \quad \text { (except for } m=y\right) .
$$

These conditions simplify the KSE drastically:

$$
\begin{aligned}
& \delta \psi_{1,2,8} \Rightarrow \Pi_{1} \epsilon \equiv\left[-e^{-c_{1}} \Gamma^{8}+e^{2 c_{1}+h_{2}} c_{1}^{\prime} \Gamma^{9}\right. \\
& \left.+\frac{1}{6} e^{5 c_{1}-2 h_{1}} \Gamma^{97}\left(e^{-2 h_{3}} k_{6,1} \Gamma^{34}+e^{2 h_{3}} k_{6,2} \Gamma^{56}\right)-\frac{1}{6} e^{8 c_{1}-4 h_{1}} k_{7}\right] \epsilon=0, \\
& \Gamma^{3} \delta \psi_{3}+\Gamma^{5} \delta \psi_{5} \Rightarrow \Pi_{2} \epsilon \equiv\left[2 e^{2 c_{1}+h_{2}}\left(-2 c_{1}^{\prime}+h_{1}^{\prime}\right) \Gamma^{9}+\frac{1}{2} e^{5 c_{1}-2 h_{1}+h_{2}} \Gamma^{7}\left(e^{-2 h_{3}} \Gamma^{34}+e^{+2 h_{3}} \Gamma^{56}\right)\right. \\
& \left.-\frac{1}{6} e^{5 c_{1}-2 h_{1}} \Gamma^{97}\left(e^{-2 h_{3}} k_{6,1} \Gamma^{34}+e^{2 h_{3}} k_{6,2} \Gamma^{56}\right)+\frac{2}{3} e^{8 c_{1}-4 h_{1}} k_{7}\right] \epsilon=0, \\
& \Gamma^{3} \delta \psi_{3}-\Gamma^{5} \delta \psi_{5} \Rightarrow \Pi_{3} \epsilon \equiv\left[2 e^{2 c_{1}+h_{2}} h_{3}^{\prime} \Gamma^{9}+\frac{1}{2} e^{5 c_{1}-2 h_{1}+h_{2}} \Gamma^{7}\left(e^{-2 h_{3}} \Gamma^{34}-e^{2 h_{3}} \Gamma^{56}\right)\right. \\
& \left.-\frac{1}{2} e^{5 c_{1}-2 h_{1}} \Gamma^{97}\left(e^{-2 h_{3}} k_{6,1} \Gamma^{34}-e^{2 h_{3}} k_{6,2} \Gamma^{56}\right)\right] \epsilon=0, \\
& \delta \psi_{7} \Rightarrow \Pi_{4} \epsilon \equiv\left[e^{2 c_{1}+h_{2}}\left(c_{1}^{\prime}+h_{2}^{\prime}\right) \Gamma^{9}+\frac{3}{2} e^{-c_{1}-h_{2}} \Gamma^{7}\left(\Gamma^{34}+\Gamma^{56}\right)\right. \\
& -\frac{1}{2} e^{5 c_{1}-2 h_{1}+h_{2}} \Gamma^{7}\left(e^{-2 h_{3}} \Gamma^{34}+e^{2 h_{3}} \Gamma^{56}\right) \\
& \left.-\frac{1}{3} e^{5 c_{1}-2 h_{1}} \Gamma^{97}\left(e^{-2 h_{3}} k_{6,1} \Gamma^{34}+e^{2 h_{3}} k_{6,2} \Gamma^{56}\right)-\frac{1}{6} e^{8 c_{1}-4 h_{1}} k_{7}\right] \epsilon=0 \text {, } \\
& \delta \psi_{9} \Rightarrow \Pi_{5} \epsilon \equiv\left[2 e^{2 c_{1}+h_{2}} \Gamma^{9} \partial_{y}+e^{2 c_{1}+h_{2}} c_{1}^{\prime} \Gamma^{9}\right. \\
& \left.-\frac{1}{3} e^{5 c_{1}-2 h_{1}} \Gamma^{97}\left(e^{-2 h_{3}} k_{6,1} \Gamma^{34}+e^{2 h_{3}} k_{6,2} \Gamma^{56}\right)-\frac{1}{6} e^{8 c_{1}-4 h_{1}} k_{7}\right] \epsilon=0, \\
& \delta \psi_{+} \Rightarrow \Pi_{6} \epsilon \equiv\left[\frac{1}{2} \mathbf{F}_{+}+\frac{3}{4} e^{c_{1}} c_{3}^{\prime} \Gamma^{97}\right. \\
& \left.+\frac{3}{4} e^{4 c_{1}-2 h_{1}}\left(e^{-2 h_{3}}\left(1+c_{3}\right) \Gamma^{34}-e^{2 h_{3}}\left(1-c_{3}\right) \Gamma^{56}\right)\right] \epsilon=0 .
\end{aligned}
$$

The last equation, $\delta \psi_{-}=0$, is equivalent to $\mathfrak{L}_{M} \epsilon=0$. 
Computation of $\Omega$ and determination of $\left(e^{9^{\prime}}\right)$ We can use the projection conditions to compute various components of $\Omega$. Assuming for now that $k_{7} \neq 0$, we find

$$
\begin{aligned}
\bar{\epsilon} \Gamma_{+78}\left(\Pi_{1}+\Pi_{2}\right) \epsilon=0 \Rightarrow \Omega_{+7}=0, \\
\bar{\epsilon} \Gamma_{+9} \Pi_{1} \epsilon=0 \Rightarrow \Omega_{+9}=\frac{6 c_{1}^{\prime}}{k_{7}} e^{-6 c_{1}+4 h_{1}+h_{2}}, \\
\bar{\epsilon} \Gamma_{+}\left(3 \Pi_{1}+\Pi_{2}+\Pi_{4}\right) \epsilon=0 \Rightarrow \Omega_{+8}=\frac{2 c_{1}^{\prime}}{k_{7}}\left(2 h_{1}^{\prime}+h_{2}^{\prime}\right) e^{-3 c_{1}+4 h_{1}+2 h_{2}} .
\end{aligned}
$$

It is also easy to show that $\Omega_{+i}=0(i=1, \cdots, 6)$ in a similar way. Thus, we arrive at the canonical form of $\Omega=e^{+} \wedge e^{9^{\prime}}$ upon the following rotation among vielbein:

$$
\left(\begin{array}{l}
e^{8^{\prime}} \\
e^{9^{\prime}}
\end{array}\right)=\left(\begin{array}{cc}
\cos \zeta & \sin \zeta \\
-\sin \zeta & \cos \zeta
\end{array}\right)\left(\begin{array}{l}
e^{8} \\
e^{9}
\end{array}\right)
$$

where

$$
\cos \zeta=\frac{6 c_{1}^{\prime}}{k_{7}} e^{-6 c_{1}+4 h_{1}+h_{2}}, \quad \sin \zeta=-\frac{2 c_{1}^{\prime}}{k_{7}}\left(2 h_{1}^{\prime}+h_{2}^{\prime}\right) e^{-3 c_{1}+4 h_{1}+2 h_{2}} .
$$

From $\cos ^{2} \zeta+\sin ^{2} \zeta=1$, we find a non-trivial condition among the unknown functions,

$$
4\left(c_{1}^{\prime}\right)^{2} e^{-12 c_{1}+8 h_{1}+2 h_{2}}\left[9+\left(2 h_{1}^{\prime}+h_{2}^{\prime}\right)^{2} e^{6 c_{1}+2 h_{2}}\right]=k_{7}^{2} .
$$

Another non-trivial relation follows from

$$
\bar{\epsilon} \Gamma_{+}\left(2 \Pi_{2}-\Pi_{4}\right) \epsilon=0 \Rightarrow k_{7}^{2}=4 c_{1}^{\prime}\left(9 c_{1}^{\prime}-4 h_{1}^{\prime}+h_{2}^{\prime}\right) e^{-12 c_{1}+8 h_{1}+2 h_{2}},
$$

where we used the form of $\Omega_{+9}$ in (4.18). We can eliminate $k_{7}$ from the two equations above to obtain a relation among metric components only

$$
4 h_{1}^{\prime}-h_{2}^{\prime}=-c_{1}^{\prime}\left(2 h_{1}^{\prime}+h_{2}^{\prime}\right)^{2} e^{6 c_{1}+2 h_{2}} .
$$

Computation of $\Sigma$ and confirmation of $\left(e^{9^{\prime}}\right)$ We can also use the projection conditions to compute components of $\Sigma$. For instance, we find

$$
\begin{aligned}
& \bar{\epsilon} \Gamma_{+9}\left(\Pi_{2}+\Pi_{3}\right) \epsilon=0 \Rightarrow \Sigma_{+7934}=2 e^{-3 c_{1}+2 h_{1}+2 h_{3}}\left(h_{1}^{\prime}+h_{3}^{\prime}\right), \\
& \bar{\epsilon} \Gamma_{+9}\left(\Pi_{2}-\Pi_{3}\right) \epsilon=0 \Rightarrow \Sigma_{+7956}=2 e^{-3 c_{1}+2 h_{1}-2 h_{3}}\left(h_{1}^{\prime}-h_{3}^{\prime}\right),
\end{aligned}
$$

It follows from $\Sigma_{+i j 34}=\Sigma_{+i j 56}$ that

$$
h_{1}^{\prime} \sinh \left(2 h_{3}\right)+h_{3}^{\prime} \cosh \left(2 h_{3}\right)=0 .
$$

Next, we can determine $\left\{k_{6, a}\right\}$. Note that

$$
\begin{aligned}
& \bar{\epsilon} \Gamma_{+}\left(3 \Pi_{2}+\Pi_{3}\right) \epsilon=0 \Rightarrow k_{6,1} \Sigma_{+7934}=-\frac{4 c_{1}^{\prime}}{k_{7}}\left(h_{1}^{\prime}+2 h_{2}^{\prime}+3 h_{3}^{\prime}\right) e^{-9 c_{1}+6 h_{1}+2 h_{2}+2 h_{3}}, \\
& \bar{\epsilon} \Gamma_{+}\left(3 \Pi_{2}-\Pi_{3}\right) \epsilon=0 \Rightarrow k_{6,2} \Sigma_{+7956}=-\frac{4 c_{1}^{\prime}}{k_{7}}\left(h_{1}^{\prime}+2 h_{2}^{\prime}-3 h_{3}^{\prime}\right) e^{-9 c_{1}+6 h_{1}+2 h_{2}-2 h_{3}} .
\end{aligned}
$$


Then, using (4.24), we find

$$
\begin{aligned}
& k_{6,1}=-\frac{2 c_{1}^{\prime}\left(h_{1}^{\prime}+2 h_{2}^{\prime}+3 h_{3}^{\prime}\right)}{k_{7}\left(h_{1}^{\prime}+h_{3}^{\prime}\right)} e^{-6 c_{1}+4 h_{1}+2 h_{2}}, \\
& k_{6,2}=-\frac{2 c_{1}^{\prime}\left(h_{1}^{\prime}+2 h_{2}^{\prime}-3 h_{3}^{\prime}\right)}{k_{7}\left(h_{1}^{\prime}-h_{3}^{\prime}\right)} e^{-6 c_{1}+4 h_{1}+2 h_{2}} .
\end{aligned}
$$

From $\Sigma=e^{+} \wedge \Phi$ with the rotation taken into account, we deduce

$$
\begin{aligned}
& \Sigma_{+7934}=-\sin \zeta \\
\Rightarrow \quad & 2 e^{2 h_{3}}\left(h_{1}^{\prime}+h_{3}^{\prime}\right)=\frac{2 c_{1}^{\prime}}{k_{7}}\left(2 h_{1}^{\prime}+h_{2}^{\prime}\right) e^{2 h_{1}+2 h_{2}} .
\end{aligned}
$$

Constancy of spinor and further relations Recall that the canonical G-structure frame (where $\Omega=e^{+} \wedge e^{9^{\prime}}$ holds) is related to our original frame (2.39) by the rotation (4.19). Since the projection conditions are mapped to each other by

$$
\left(-\sin \zeta \Gamma^{8}+\cos \zeta \Gamma^{9}\right) \epsilon=\epsilon \quad \Gamma^{9} \epsilon^{\prime}=\epsilon^{\prime},
$$

the Killing spinors in the two frames should be related by

$$
\epsilon^{\prime}=\exp \left[(\zeta / 2) \Gamma^{89}\right] \epsilon=\left[\cos (\zeta / 2)+\Gamma^{89} \sin (\zeta / 2)\right] \epsilon
$$

We can now take advantage of another important result of [29] that $\epsilon^{\prime}$ is a constant spinor. Plugging (4.30) this into the $\delta \psi_{9}=0$ condition in (4.17) and using $d \epsilon^{\prime}=0$, we obtain

$$
\begin{array}{r}
\Pi_{5} \epsilon=\left[\epsilon^{2 c_{1}+h_{2}} \zeta^{\prime} \Gamma^{8}+e^{2 c_{1}+h_{2}} c_{1}^{\prime} \Gamma^{9}-\frac{1}{3} e^{5 c_{1}-2 h_{1}} \Gamma^{97}\left(e^{-2 h_{3}} k_{6,1} \Gamma^{34}+e^{2 h_{3}} k_{6,2} \Gamma^{56}\right)\right. \\
\left.-\frac{1}{6} e^{8 c_{1}-4 h_{1}} k_{7}\right] \epsilon=0,
\end{array}
$$

which further implies

$$
\begin{aligned}
\Pi_{1}^{\prime} \epsilon & \equiv\left(\Pi_{1}-\Pi_{5}\right) \epsilon \\
& =\left[\left(-\zeta^{\prime} e^{2 c_{1}+h_{2}}-e^{-c_{1}}\right) \Gamma^{8}+\frac{1}{2} e^{5 c_{1}-2 h_{1}} \Gamma^{97}\left(e^{-2 h_{3}} k_{6,1} \Gamma^{34}+e^{2 h_{3}} k_{6,2} \Gamma^{56}\right)\right] \epsilon=0, \\
\Pi_{4}^{\prime} \epsilon & \equiv\left(\Pi_{4}-\Pi_{5}\right) \epsilon=\left[-\zeta^{\prime} e^{2 c_{1}+h_{2}} \Gamma^{8}+h_{2}^{\prime} e^{2 c_{1}+h_{2}} \Gamma^{9}+\frac{3}{2} e^{-c_{1}-h_{2}} \Gamma^{7}\left(\Gamma^{34}+\Gamma^{56}\right)\right. \\
\Pi_{0} \epsilon & \left.\equiv-\frac{1}{2} e^{5 c_{1}-2 h_{1}+h_{2}} \Gamma^{7}\left(e^{-2 h_{3}} \Gamma^{34}+e^{2 h_{3}} \Gamma^{56}\right)\right] \epsilon=0, \\
& =\frac{1}{2}\left[1+\frac{2}{k_{7}} e^{-6 c_{1}+4 h_{1}}\left(2 \Pi_{1}+\Pi_{5}\right) \epsilon \quad\left(\left(-\zeta^{\prime} e^{h_{2}}+2 e^{-3 c_{1}}\right) \Gamma^{8}-3 c_{1}^{\prime} e^{h_{2}} \Gamma^{9}\right)\right] \epsilon=0 .
\end{aligned}
$$

Comparing $\Pi_{0}$ with 4.20 ) and $(4.29)$, we make the identification,

$$
\sin \zeta=\frac{2}{k_{7}}\left(-\zeta^{\prime} e^{h_{2}}+2 e^{-3 c_{1}}\right) e^{-6 c_{1}+4 h_{1}} .
$$


Next, we have

$$
\bar{\epsilon} \Gamma_{+} \Pi_{4}^{\prime} \epsilon=0 \Rightarrow \zeta^{\prime} \sin \zeta=-h_{2}^{\prime} \cos \zeta .
$$

Integrating it and using (4.20) again, we find

$$
\cos \zeta=\kappa e^{h_{2}}=\frac{6 c_{1}^{\prime}}{k_{7}} e^{-6 c_{1}+4 h_{1}+h_{2}} \Rightarrow k_{7}=\frac{6 c_{1}^{\prime}}{\kappa} e^{-6 c_{1}+4 h_{1}},
$$

where $\kappa$ is an integration constant. Combining it with (4.22), we also find

$$
9 c_{1}^{\prime} e^{-2 h_{2}}=\kappa\left(9 c_{1}^{\prime}-4 h_{1}^{\prime}+h_{2}^{\prime}\right) .
$$

As a consistency check, we combine (4.20), (4.33) and (4.34) to find

$$
4 h_{1}^{\prime}-h_{2}^{\prime}=-c_{1}^{\prime}\left(2 h_{1}^{\prime}+h_{2}^{\prime}\right)^{2} e^{6 c_{1}+2 h_{2}},
$$

which is identical to (4.23).

Combining $\Pi_{1}^{\prime}$ with 4.27 , (4.33) and 4.35) gives more projection conditions and constraints on unknown functions

$$
\Pi_{1}^{\prime} \epsilon=0 \Rightarrow \Gamma^{34789} \epsilon=-\epsilon, \quad 2 h_{1}^{\prime}+h_{2}^{\prime}=6 \kappa\left(h_{1}^{\prime}+h_{3}^{\prime}\right) e^{-6 c_{1}+2 h_{1}-2 h_{2}+2 h_{3}} .
$$

and we can easily check $\Pi_{2,3}$ conditions are automatically satisfied with (4.38). The remaining projection conditions, $\Pi_{4}^{\prime}, \Pi_{6}$, produce the following conditions

$$
\begin{aligned}
\Pi_{4}^{\prime} \epsilon=0 \Rightarrow \kappa & =1 \\
\Pi_{6} \epsilon=0 \Rightarrow+ & \sin \zeta\left(e^{-2 c_{1}} k_{3}+3 e^{4 c_{1}-2 h_{1}}\left(c_{3} \cosh \left(2 h_{3}\right)-\sinh \left(2 h_{3}\right)\right)\right) \\
= & \frac{3}{2} c_{3}^{\prime} e^{c_{1}}+e^{-3 c_{1}}\left(e^{-2 c_{1}} k_{1}+e^{4 c_{1}-2 h_{1}}\left(e^{-2 h_{3}} k_{4,1}+e^{2 h_{3}} k_{4,2}\right)\right), \\
- & \cos \zeta\left(e^{-2 c_{1}} k_{3}+3 e^{4 c_{1}-2 h_{1}}\left(c_{3} \cosh \left(2 h_{3}\right)-\sinh \left(2 h_{3}\right)\right)\right) \\
& =e^{h_{2}}\left(e^{-2 c_{1}} k_{2}+e^{4 c_{1}-2 h_{1}}\left(e^{-2 h_{3}} k_{5,1}+e^{2 h_{3}} k_{5,2}\right)\right) .
\end{aligned}
$$

Summary We have found that the null Killing spinor equations impose several coupled equations for the unknown functions $\left\{c_{1}, h_{1}, h_{2}, h_{3}, k_{6,1}, k_{6,2}, k_{7}\right\}$. The independent equations can be summarized as follows:

$$
\begin{aligned}
& 4 h_{1}^{\prime}-h_{2}^{\prime}=-c_{1}^{\prime}\left(2 h_{1}^{\prime}+h_{2}^{\prime}\right)^{2} e^{6 c_{1}+2 h_{2},} \\
& 9 c_{1}^{\prime}=\left(9 c_{1}^{\prime}-4 h_{1}^{\prime}+h_{2}^{\prime}\right) e^{2 h_{2},}, \\
& 2 h_{1}^{\prime}+h_{2}^{\prime}=6\left(h_{1}^{\prime}+h_{3}^{\prime}\right) e^{-6 c_{1}+2 h_{1}-2 h_{2}+2 h_{3},} \\
& h_{3}^{\prime} \cosh \left(2 h_{3}\right)=-h_{1}^{\prime} \sinh \left(2 h_{3}\right), \\
& k_{6,1}=-\frac{h_{1}^{\prime}+2 h_{2}^{\prime}+3 h_{3}^{\prime}}{3\left(h_{1}^{\prime}+h_{3}^{\prime}\right)} e^{2 h_{2},} \\
& k_{6,2}=-\frac{h_{1}^{\prime}+2 h_{2}^{\prime}-3 h_{3}^{\prime}}{3\left(h_{1}^{\prime}-h_{3}^{\prime}\right)} e^{2 h_{2}}, \\
& k_{7}=6 c_{1}^{\prime} e^{-6 c_{1}+4 h_{1}} .
\end{aligned}
$$


The first four equations were obtained in (4.23), (4.36), (4.38) and (4.25), respectively; recall also (4.39). They give Block $\mathrm{A}$ in section 3. The auxiliary equations in Block A concerning $\cos \zeta$ and $\sin \zeta$ come from combinations of (4.35), (4.39), (4.20) and (4.33). The equations for $\left(k_{6,1}, k_{6,2}, k_{7}\right)$ were obtained in (4.27) and (4.35). They give the last three entries of Block $\mathrm{C}$ in section 3 .

\subsection{Dynamical supercharges and time-like Killing spinor}

G-structure The commutation relation $\{\bar{Q}, Q\}=H$ implies that an $\mathcal{N}=2$ superSchrödinger geometry should admit a time-like Killing spinor. The general study of geometries admitting a single time-like Killing spinor has been done in [28]. The metric takes the form

$$
d s^{2}=-\Delta^{2}(d t+\omega)^{2}+\Delta^{-1} g_{m n} d x^{m} d x^{n}
$$

The base manifold $\mathcal{B}$ with metric $g_{m n}$ is orthogonal to time direction and has $S U(5)$ structure instead of $\operatorname{Spin}(7)$. The $S U(5)$ structure is given by a pair of spinors $\left(\epsilon_{\bar{Q}}, \epsilon_{Q}\right)$.

$$
\begin{aligned}
\epsilon_{d} & \equiv \frac{1}{\sqrt{2}}\left(\epsilon_{\bar{Q}}+\epsilon_{Q}\right) \\
K & =\bar{\epsilon}_{d} \Gamma_{a} \epsilon_{d} e^{a}=\Delta^{2}(d t+\omega) \\
\Omega & =\bar{\epsilon}_{d} \Gamma_{a b} \epsilon_{d} e^{a b}
\end{aligned}
$$

Here, $K$ is the dual one-form of $H$ and $\Omega$ is the Kähler form of the base manifold $B$. We can always decompose $\epsilon$ by the eigenvalue of $\Gamma^{+-}$and find the relation with the kinematical supercharges

$$
\begin{aligned}
& \epsilon_{d}=\frac{e^{c_{1}}}{r}\left(\Gamma^{+} \eta_{1}+\eta_{2}\right) \\
& \epsilon_{k} \equiv \frac{1}{2}\left(\epsilon_{q}+\epsilon_{\bar{q}}\right)=\frac{1}{\sqrt{2}} \Gamma^{+1} \eta_{2},
\end{aligned}
$$

where

$$
\begin{aligned}
& \Gamma^{-} \eta_{i}=0, \quad \Gamma^{3456} \eta_{i}=-\eta_{i}, \quad(i=1,2) \\
& \bar{\eta}_{2} \Gamma_{-} \eta_{2}=-1, \quad \bar{\eta}_{1} \Gamma_{-} \eta_{1}=-\frac{1}{4} c_{2} e^{-2 c_{1}} .
\end{aligned}
$$

The last two relations are derived from the relation of the spinor bilinear $K$. The $\eta_{i}$ 's are orthogonal to each other and have zero-norm $\bar{\eta}_{i} \eta_{j}=0$.

Killing spinor equations From the results of the previous subsection, we already have some information about the dynamical supercharge

$$
\left.\Gamma^{3456} \epsilon_{d}=-\epsilon_{d}, \quad r \partial_{r} \epsilon_{d}=-\epsilon_{d}, \quad \partial_{m} \epsilon_{d}=0 \quad \text { (except for } m=y, r\right)
$$


The Killing spinor equations for the component spinors $\eta_{i}$ are given by

- $\delta \psi_{1,2}$

$\Rightarrow\left[e^{-c_{1}} \Gamma^{8}-e^{2 c_{1}+h_{2}} c_{1}^{\prime} \Gamma^{9}+\frac{1}{6} e^{5 c_{1}-2 h_{1}} \Gamma^{97}\left(e^{-2 h_{3}} k_{6,1} \Gamma^{34}+e^{2 h_{2}} k_{6,2} \Gamma^{56}\right)-e^{8 c_{1}-4 h_{1}} \frac{1}{6} k_{7}\right] \eta_{1}$

$+\frac{1}{6}\left[\mathbf{F}_{+}-\frac{3}{2} \Gamma^{-1} \mathbf{F}_{1}\right] \eta_{2}=0$

- $\Gamma^{3} \delta \psi_{3}+\Gamma^{5} \delta \psi_{5}$

$$
\begin{gathered}
\Rightarrow\left[-2\left(-2 c_{1}^{\prime}+h_{1}^{\prime}\right) e^{2 c_{1}+h_{2}} \Gamma^{9}-\frac{1}{2} e^{5 c_{1}-2 h_{1}+h_{2}} \Gamma^{7}\left(e^{-2 h_{3}} \Gamma^{34}+e^{2 h_{3}} \Gamma^{56}\right)\right. \\
\left.-\frac{1}{6} e^{5 c_{1}-2 h_{1}} \Gamma^{97}\left(e^{-2 h_{3}} k_{6,1} \Gamma^{34}+e^{2 h_{3}} k_{6,2} \Gamma^{56}\right)+\frac{2}{3} e^{8 c_{1}-4 h_{1}} k_{7}\right] \eta_{1} \\
+\left[\frac{3}{4} e^{4 c_{1}-2 h_{1}}\left(e^{-2 h_{3}}\left(1+c_{3}\right) \Gamma^{34}-e^{2 h_{3}}\left(1-c_{3}\right) \Gamma^{56}\right)\right. \\
+\frac{1}{6}\left(2 \mathbf{F}_{+}-\frac{3}{2}\left(\Gamma^{-3} \mathbf{F}_{3}+\Gamma^{-5} \mathbf{F}_{5}\right)\right] \eta_{2}=0,
\end{gathered}
$$

- $\Gamma^{3} \delta \psi_{3}-\Gamma^{5} \delta \psi_{5}$

$$
\begin{aligned}
\Rightarrow[- & 2 h_{3}^{\prime} e^{2 c_{1}+h_{2}} \Gamma^{9}-\frac{1}{2} e^{5 c_{1}-2 h_{1}+h_{2}} \Gamma^{7}\left(e^{-2 h_{3}} \Gamma^{34}-e^{2 h_{3}} \Gamma^{56}\right) \\
& \left.-\frac{1}{2} e^{5 c_{1}-2 h_{1}} \Gamma^{97}\left(e^{-2 h_{3}} k_{6,1} \Gamma^{34}-e^{2 h_{3}} k_{6,2} \Gamma^{56}\right)\right] \eta_{1} \\
& +\left[\frac{3}{4} e^{4 c_{1}-2 h_{1}}\left(e^{-2 h_{3}}\left(1+c_{3}\right) \Gamma^{34}+e^{2 h_{3}}\left(1-c_{3}\right) \Gamma^{56}\right)-\frac{1}{4}\left(\Gamma^{-3} \mathbf{F}_{3}-\Gamma^{-5} \mathbf{F}_{5}\right)\right] \eta_{2}=0,
\end{aligned}
$$

- $\delta \psi_{7}$

$$
\begin{gathered}
\Rightarrow\left[-e^{2 c_{1}+h_{2}}\left(c_{1}^{\prime}+h_{2}^{\prime}\right) \Gamma^{9}-\frac{3}{2} e^{-c_{1}-h_{2}} \Gamma^{7}\left(\Gamma^{34}+\Gamma^{56}\right)+\frac{1}{2} e^{5 c_{1}-2 h_{1}+h_{2}} \Gamma^{7}\left(e^{-2 h_{3}} \Gamma^{34}+e^{2 h_{3}} \Gamma^{56}\right)\right. \\
\left.-\frac{1}{3} e^{5 c_{1}-2 h_{1}} \Gamma^{97}\left(e^{-2 h_{3}} k_{6,1} \Gamma^{34}+e^{2 h_{3}} k_{6,2} \Gamma^{56}\right)-\frac{1}{6} e^{8 c_{1}-4 h_{1}} k_{7}\right] \eta_{1} \\
+\left[-\frac{3}{4} e^{c_{1}} c_{3}^{\prime} \Gamma^{79}+\frac{1}{6}\left(\mathbf{F}_{+}-\frac{3}{2} \Gamma^{-7} \mathbf{F}_{7}\right)\right] \eta_{2}=0,
\end{gathered}
$$

- $\delta \psi_{8}$

$\Rightarrow\left[3 e^{-c_{1}} \Gamma^{8}-e^{2 c_{1}+h_{2}} c_{1}^{\prime} \Gamma^{9}+\frac{1}{6} e^{5 c_{1}-2 h_{1}} \Gamma^{97}\left(e^{-2 h_{3}} k_{6,1} \Gamma^{34}+e^{2 h_{3}} k_{6,2} \Gamma^{56}\right)-\frac{1}{6} e^{8 c_{1}-4 h_{1}} k_{7}\right] \eta_{1}$

$$
+\frac{1}{6}\left[\mathbf{F}_{+}-\frac{3}{2} \Gamma^{-8} \mathbf{F}_{8}\right] \eta_{2}=0 \text {, }
$$

- $\delta \psi_{9}$

$\Rightarrow\left[-2 e^{2 c_{1}+h_{2}} \Gamma^{9} \partial_{y}-3 e^{2 c_{1}+h_{2}} c_{1}^{\prime} \Gamma^{9}\right.$

$$
\begin{aligned}
\left.-\frac{1}{3} e^{5 c_{1}-2 h_{1}} \Gamma^{97}\left(e^{-2 h_{3}} k_{6,1} \Gamma^{34}+e^{2 h_{3}} k_{6,2} \Gamma^{56}\right)-\frac{1}{6} e^{8 c_{1}-4 h_{1}} k_{7}\right] \eta_{1} \\
+\left[-\frac{3}{4} e^{c_{1}} c_{3}^{\prime} \Gamma^{79}+\frac{1}{6}\left(\mathbf{F}_{+}-\frac{3}{2} \Gamma^{-9} \mathbf{F}_{9}\right)\right] \eta_{2}=0,
\end{aligned}
$$

- $\delta \psi_{+}$

$$
\begin{aligned}
\Rightarrow\left[\frac{3}{4} e^{c_{1}} c_{3}^{\prime} \Gamma^{79}-\frac{3}{4} e^{4 c_{1}-2 h_{1}}\left(e^{-2 h_{3}}\left(1+c_{3}\right) \Gamma^{34}\right.\right. & \left.\left.-e^{2 h_{3}}\left(1-c_{3}\right) \Gamma^{56}\right)+\frac{1}{2} \mathbf{F}_{+}\right] \eta_{1} \\
& +\left[c_{2} e^{-3 c_{1}} \Gamma^{8}-\frac{1}{2} c_{2}^{\prime} e^{h_{2}} \Gamma^{9}\right] \eta_{2}=0 .
\end{aligned}
$$

The equations for $\delta \psi_{1}, \delta \psi_{8}$ give a relation between $\eta_{1}$ and $\eta_{2}$,

$$
\eta_{1}=\frac{1}{4}\left(e^{2 c_{1}-2 h_{1}}\left(e^{-2 h_{3}} k_{4,1}+e^{2 h_{3}} k_{4,2}\right) \Gamma^{34}-e^{-c_{1}} k_{3} \Gamma^{79}-e^{-c_{1}+h_{2}} k_{2} \Gamma^{1289}\right) \eta_{2} .
$$


Sufficiency of Killing spinor equations In general, Killing spinor equations do not restrict every single component of the metric and flux. To determine all components, we must supplement the Killing spinor equations with some components of the equation of motion. However, the situation is better for our problem. Note that we have the time-like Killing vector $H$ as well as the null Killing vector $M$. In addition, our flux does not have components along the $e^{-}$direction. These facts together imply that in our case, the Killing spinor equations are sufficient to determine all components of the metric and flux [28].

Computation of $\Omega$ and further constraints We wrote the dynamical Killing spinor in terms of $\eta_{2}$ which inherit the properties of the kinematical Killing spinor $\epsilon_{k}$. The projection conditions on $\eta_{2}$ are summarized by

$$
\begin{aligned}
& \Gamma_{-} \eta_{2}=0, \quad \Gamma^{3456} \eta_{2}=-\eta_{2}, \quad \Gamma^{34789} \eta_{2}=-\eta_{2}, \\
& \left(-\sin \zeta \Gamma^{8}+\cos \zeta \Gamma^{9}\right) \eta_{2}=\eta_{2} .
\end{aligned}
$$

Using these and the relations (4.43), we can derive the explicit form of the Kähler form $\Omega$

$$
\begin{aligned}
\Omega=\frac{1}{2 r^{2}}[ & -\left(c_{2} e^{+}+2 e^{2 c_{1}} e^{-}\right) e^{9^{\prime}} \\
& +\left(e^{4 c_{1}-2 h_{1}}\left(e^{-2 h_{3}} k_{4,1}+e^{2 h_{3}} k_{4,2}\right)-e^{c_{1}} k_{3} \sin \zeta\right)\left(-e^{12}+e^{34}+e^{56}\right) \\
& +\left(e^{4 c_{1}-2 h_{1}}\left(e^{-2 h_{3}} k_{4,1}+e^{2 h_{3}} k_{4,2}\right) \cos \zeta+e^{c_{1}+h_{2}} k_{2} \sin \zeta\right) e^{78} \\
& \left.+\left(e^{4 c_{1}-2 h_{1}}\left(e^{-2 h_{3}} k_{4,1}+e^{2 h_{3}} k_{4,2}\right) \sin \zeta-e^{c_{1}+h_{2}} k_{2} \cos \zeta-e^{c_{1}} k_{3}\right) e^{79}\right] .
\end{aligned}
$$

We used the condition $i_{K} \Omega=0$ to derive $\Omega_{+a}$ components. The G-structure equations give further relations

$$
\begin{aligned}
& \Omega_{a}{ }^{c} \Omega_{c}{ }^{b}=-K_{a} K^{b}+\delta_{a}^{b} K^{2} \\
& \Rightarrow \quad k_{2}=-k_{3}, \quad c_{2}=\frac{1}{4}\left(e^{3 c_{1}-2 h_{1}}\left(e^{-2 h_{3}} k_{4,1}+e^{2 h_{3}} k_{4,2}\right)-k_{3} \sin \zeta\right)^{2} .
\end{aligned}
$$

From $d \Omega=i_{K} F$,

$$
\begin{aligned}
k_{1} & =-2\left[e^{6 c_{1}-2 h_{1}}\left(e^{-2 h_{3}} k_{4,1}+e^{2 h_{3}} k_{4,2}\right)-e^{3 c_{1}} k_{3} \sin \zeta\right], \\
k_{3} & =-\frac{3}{2} c_{3}^{\prime} e^{3 c_{1}} \sin \zeta+\frac{1}{2} e^{-3 c_{1}} k_{1} \sin \zeta+\frac{1}{4} \partial_{y}\left(e^{2 h_{2}} k_{1}\right), \\
k_{4,1} & =\frac{3}{2}\left[-\left(c_{3}+1\right) e^{3 c_{1}} \sin \zeta-\frac{1}{6} k_{1}\left(2 e^{-6 c_{1}+2 h_{1}+2 h_{3}}-e^{2 h_{2}}\right)\right], \\
k_{4,2} & =\frac{3}{2}\left[-\left(c_{3}-1\right) e^{3 c_{1}} \sin \zeta-\frac{1}{6} k_{1}\left(2 e^{-6 c_{1}+2 h_{1}-2 h_{3}}-e^{2 h_{2}}\right)\right], \\
k_{5,1} & =\frac{1}{4}\left[6\left(c_{3}+1\right)+e^{-3 c_{1}} \sin \zeta+\partial_{y}\left(e^{-6 c_{1}+2 h_{1}+2 h_{3}} k_{1}\right)\right], \\
k_{5,1} & =\frac{1}{4}\left[6\left(c_{3}-1\right)+e^{-3 c_{1}} \sin \zeta+\partial_{y}\left(e^{-6 c_{1}+2 h_{1}-2 h_{3}} k_{1}\right)\right] .
\end{aligned}
$$

We can use the Bianchi identity of $k_{1}^{\prime}=-4 k_{2}$ and (3.25), (4.35) to simplify the equations for $k_{3}, k_{5, i}$ such that

$$
\begin{aligned}
& 3 c_{3}^{\prime}=2\left(k_{1} e^{-6 c_{1}} \frac{h_{1}^{\prime}-h_{2}^{\prime}}{2 h_{1}^{\prime}+h_{2}^{\prime}}+k_{3} e^{-3 c_{1}} \sin \zeta\right), \\
& e^{-2 h_{3}} k_{5,1}-e^{2 h_{3}} k_{5,2}=-3\left(c_{3} \sinh \left(2 h_{3}\right)-\cosh \left(2 h_{3}\right)\right) .
\end{aligned}
$$


By combining these results, we can further reduce the equation (4.40) to a simpler form,

$$
\begin{aligned}
& k_{5,1}=-\frac{3}{2}\left(c_{3}-1\right), \quad k_{5,2}=-\frac{3}{2}\left(c_{3}+1\right), \\
& 3 c_{1}^{\prime}+k_{1} e^{-6 c_{1}}=6 \sin \zeta\left(c_{3} \cosh \left(2 h_{3}\right)-\sinh \left(2 h_{3}\right)\right) e^{3 c_{1}-2 h_{1}} .
\end{aligned}
$$

It is straightforward to show that the solutions which satisfy all the equations we have found so far will also satisfy the rest of Killing spinor equations.

Summary We can find one more relation for $k_{1}$ and $c_{3}$ from the super-Schrödinger algebra; our solution for the Killing spinor realizes the commutation relation (2.26) if

$$
k_{1}=-\frac{6 c_{3}}{\sin \zeta} e^{3 c_{1}}
$$

Thus we have found all equations in Block B,

$$
\begin{aligned}
& k_{2}=-k_{3}, \\
& k_{1}=-\frac{6 c_{3}}{\sin \zeta} e^{3 c_{1}}, \\
& 3 c_{1}^{\prime}+k_{1} e^{-6 c_{1}}=6 \sin \zeta\left(c_{3} \cosh \left(2 h_{3}\right)-\sinh \left(2 h_{3}\right)\right) e^{3 c_{1}-2 h_{1}}, \\
& 3 c_{3}^{\prime}=2\left(k_{1} e^{-6 c_{1}} \frac{h_{1}^{\prime}-h_{2}^{\prime}}{2 h_{1}^{\prime}+h_{2}^{\prime}}+k_{3} e^{-3 c_{1}} \sin \zeta\right),
\end{aligned}
$$

and the first five entries of Block $C$,

$$
\begin{aligned}
& c_{2}=\left(\frac{1}{4} k_{1} e^{-3 c_{1}}\right)^{2}, \\
& k_{4,1}=\frac{3}{2}\left[-\left(c_{3}+1\right) e^{3 c_{1}} \sin \zeta-\frac{1}{6} k_{1}\left(2 e^{-6 c_{1}+2 h_{1}+2 h_{3}}-e^{2 h_{2}}\right)\right], \\
& k_{4,2}=\frac{3}{2}\left[-\left(c_{3}-1\right) e^{3 c_{1}} \sin \zeta-\frac{1}{6} k_{1}\left(2 e^{-6 c_{1}+2 h_{1}-2 h_{3}}-e^{2 h_{2}}\right)\right], \\
& k_{5,1}=-\frac{3}{2}\left(c_{3}-1\right) \\
& k_{5,2}=-\frac{3}{2}\left(c_{3}+1\right) .
\end{aligned}
$$

The dynamical Killing spinor $\epsilon_{d}$ and Kähler form $\Omega$ is reduced to

$$
\begin{aligned}
& \epsilon_{d}=\frac{e^{c_{1}}}{r}\left(\Gamma^{+} \eta_{1}+\eta_{2}\right)=\frac{e^{c_{1}}}{r}\left(-\frac{1}{8} e^{-4 c_{1}} k_{1} \Gamma^{+34}+1\right) \eta_{2}, \\
& \Omega=-\frac{1}{2 r^{2}}\left(c_{2} e^{+}+2 e^{2 c_{1}} e^{-}\right) e^{9^{\prime}}+\frac{k_{1}}{4 r^{2}} e^{-2 c_{1}}\left(e^{12}-e^{34}-e^{56}+e^{78^{\prime}}\right) .
\end{aligned}
$$

We can see that $\Omega$ is really the Kähler form for the ten dimensional spatial manifold $\mathcal{B}$ by redefining the spatial vielbeins

$$
\Omega=\bar{e}^{12}-\bar{e}^{34}-\bar{e}^{56}+\bar{e}^{78^{\prime}}+\bar{e}^{9^{\prime} 10^{\prime}} .
$$

where

$$
\begin{aligned}
e^{i} & =\Delta^{-1 / 2} \bar{e}^{i}, \quad e^{10^{\prime}}=\Delta^{-1} \frac{1}{2 r^{2}}\left(c_{2} e^{+}+2 e^{2 c_{1}} e^{-}\right) \\
\Delta & =\left(\frac{c_{2} e^{2 c_{1}}}{r^{4}}\right)^{1 / 2}=\frac{k_{1} e^{-2 c_{1}}}{4 r^{2}}
\end{aligned}
$$




\section{5. $S^{2} \times T^{2}$ solution}

To date much of our understanding of non-relativistic geometric duals comes from work in type IIB supergravity [18-22,30-32]. A thorough account of the supersymmetry preserved appeared in [19], and a prescription was given therein to construct a special subclass of solutions based on five-dimensional Sasaki-Einstein spaces that realise the $\mathcal{N}=2$ Super-Schrödinger algebra. In this section we consider an explicit example from that class, uplift it to M-theory and comment on the Killing spinors preserved.

The general form of the solutions presented in [19] may be written as

$$
\begin{aligned}
d s^{2} & =-\frac{h d t^{2}}{r^{4}}+\frac{2 d t d \psi+d r^{2}+d \vec{x}^{2}}{r^{2}}+d s_{S E_{5^{\prime}}}^{2} \\
F_{5} & =\left(1+*_{10}\right) d x^{+} \wedge d x^{-} \wedge d x_{1} \wedge d x_{2} \wedge d\left(1 / r^{4}\right), \\
G_{3} & =d x^{+} \wedge d\left(\sigma / r^{2}\right) .
\end{aligned}
$$

Here $d s_{S E_{5}}^{2}$ denotes the metric on a five-dimensional Sasaki-Einstein space $S E_{5}$ and $\sigma$ is a complex one-form on the Calabi-Yau cone $C Y_{3}$ dual to a Killing vector on $S E_{5}$. The function $h$ is given by

$$
h=|\sigma|_{S E}^{2}+\frac{1}{2}\left(\eta_{S E}\right)^{\mu} L_{\mu}, \quad L \equiv i \mathfrak{L}_{\sigma^{*}}^{S E_{5}} \sigma,
$$

where $\eta_{S E}$ is the one-form dual to the Reeb Killing vector on $S E_{5}$ and $L$ is given in terms of the Lie-derivative with respect to the vector dual to $\sigma^{*}$.

In constructing an explicit example in this class we adopt the $S E_{5}$ metric discovered in $[15,33]$

$$
\begin{aligned}
d s_{S E_{5}}^{2}= & \frac{1-c y}{6}\left(d \theta^{2}+\sin ^{2} \theta d \phi^{2}\right)+e^{-6 \lambda} \sec ^{2} \zeta d y^{2}+\frac{1}{9} \cos ^{2} \zeta(D \beta)^{2} \\
& +e^{6 \lambda}\left(d z+\frac{a c-2 y+c y^{2}}{6\left(a-y^{2}\right)} D \beta\right)^{2}
\end{aligned}
$$

where

$$
\begin{aligned}
D \beta & =d \beta-\cos \theta d \phi, \\
e^{6 \lambda} & =\frac{2\left(a-y^{2}\right)}{1-c y}, \\
\cos ^{2} \zeta & =\frac{a-3 y^{2}+2 c y^{3}}{a-y^{2}} .
\end{aligned}
$$

In general preserving six supersymmetries requires a judicious choice for $\sigma$. As explained in [19], one requires $\sigma$ is chosen so that its exterior derivative on $C Y_{3}, d\left(\frac{\sigma}{r^{2}}\right)$, is of type $(1,1)$ and primitive ${ }^{5}$. Within these constraints, we choose $\sigma$ to be the one-form dual to the Killing vector $V_{\sigma}=\kappa_{1} \partial_{\phi}-\frac{\kappa_{2}}{6} \partial_{z}$, which is the sum of two Cartans in three on (5.3) and

\footnotetext{
${ }^{5}$ In the earlier non-supersymmetric solutions [20-22] $\sigma$ was chosen dual to the Reeb vector meaning that the exterior derivative was proportional to the Kähler two-form.
} 
$\kappa_{1}$ and $\kappa_{2}$ are arbitrary complex constants. For this choice, $L$ is zero and $h$ follows from (5.2),$h=|\sigma|_{S E}^{2}$.

By T-dualising to type IIA and uplifting this solution, we obtain a deformation of the class of warped supersymmetric $A d S_{5} \times M_{6}$ solutions with base space $M_{4}=S^{2} \times T^{2}$, originally discovered in [15]. The explicit solution has manifest Schrödinger symmetry and may be expressed as follows

$$
\begin{aligned}
d s_{11 D}^{2}= & e^{2 \lambda}\left(-\frac{\tilde{h} d t^{2}}{r^{4}}+\frac{2 d t D \psi+d r^{2}+d \vec{x}^{2}}{r^{2}}\right)+e^{2 \lambda} d s_{M_{6}}^{2}, \\
d s_{M_{6}}^{2}= & \frac{1-c y}{6}\left(d \theta^{2}+\sin ^{2} \theta d \phi^{2}\right)+e^{-6 \lambda} \sec ^{2} \zeta d y^{2}+\frac{1}{9} \cos ^{2} \zeta(D \beta)^{2}+e^{-6 \lambda}\left(d \varphi_{1}^{2}+d \varphi_{2}^{2}\right), \\
F_{4}= & -\frac{2}{9}(1-c y) d y \wedge D \beta \wedge \operatorname{Vol}\left(S^{2}\right)+d\left(\frac{a c-2 y+c y^{2}}{6\left(a-y^{2}\right)} D \beta \wedge d \varphi_{1} \wedge d \varphi_{2}\right) \\
& +d\left(\frac{\tilde{\sigma}}{r^{2}}\right) \wedge d t \wedge\left(\operatorname{Im}\left(\kappa_{1}\right) d \varphi_{1}-\operatorname{Re}\left(\kappa_{1}\right) d \varphi_{2}\right),
\end{aligned}
$$

where

$$
\begin{aligned}
\tilde{h} & =\left|\kappa_{1}\right|^{2} \tilde{\sigma}_{M_{6}}^{2}, \\
D \psi & =d \psi-\operatorname{Re}(A) d \varphi_{1}-\operatorname{Im}(A) d \varphi_{2}, \\
A & =\kappa_{1} \frac{a c-2 y-c y^{2}}{6\left(a-y^{2}\right)} \cos \theta+\frac{1}{6} \kappa_{2}, \\
\tilde{\sigma} & =-\frac{1}{9} \cos ^{2} \zeta \cos \theta D \beta+\frac{1-c y}{6} \sin ^{2} \theta d \phi .
\end{aligned}
$$

Here $\tilde{\sigma}$ is the dual one form of $\partial_{\phi}$ with respect to the metric on $M_{6}$, and one may check that when $\kappa_{1}=\kappa_{2}=0$, this reduces to the original undeformed solutions [15].

This explicit example (5.5) is supersymmetric, admitting six Killing spinors: two kinematical, two Poincaré and two superconformal Killing spinors. We now turn to detailing how it preserves these supersymmetries and what form the Killing spinors take. Since these solutions are deformations of solutions of [15] with parameters $\kappa_{1}, \kappa_{2}$, we can incorporate some of the expressions from [15] wholesale.

In calculating the Killing spinors we can write the 11D gamma matrices as

$$
\begin{aligned}
\Gamma^{a} & =\rho^{a} \otimes \gamma_{7}, \\
\Gamma^{m} & =\mathbf{1} \otimes \gamma^{m}, \\
\gamma_{7} & \equiv \gamma_{1} \ldots \gamma_{6} .
\end{aligned}
$$

where $a, b=+,-, 1,2,3$ and $m, n=1,2, \ldots, 6$ are indices on $S c h_{5}$ and $M_{6}$ respectively. 
Here we take the vielbein as

$$
\begin{aligned}
e^{+} & =\frac{e^{\lambda}}{r^{2}} d t, \quad e^{-}=e^{\lambda}\left(-\frac{h}{2 r^{2}} d t+D \psi\right), \\
e^{1} & =\frac{e^{\lambda}}{r} d x^{1}, \quad e^{2}=\frac{e^{\lambda}}{r} d x^{2}, \quad e^{3}=\frac{e^{\lambda}}{r} d r, \\
e^{4} & =e^{c_{1}}\left(\frac{1-c y}{6}\right)^{1 / 2} \sigma_{1}, \quad e^{5}=e^{c_{1}}\left(\frac{1-c y}{6}\right)^{1 / 2} \sigma_{2}, \\
e^{6} & =e^{-2 \lambda} \sec \zeta d y, \quad e^{7}=\frac{e^{\lambda}}{3} \cos \zeta D \beta, \\
e^{8} & =e^{-2 \lambda} d \varphi_{1}, \quad e^{9}=e^{-2 \lambda} d \varphi_{2} .
\end{aligned}
$$

The dynamical Killing spinors may then be written as

$$
\epsilon_{d}=\frac{\kappa_{1}}{4 r}\left[-i \frac{2}{3} \cos \zeta \cos \theta \Gamma^{+78}+\sqrt{\frac{2}{3}(1-c y)} e^{i \beta} \sin \theta \Gamma^{+58}\right] \eta+\frac{1}{r} \eta
$$

and $\eta$ is the product $\psi \otimes e^{\lambda / 2} \xi$, with $\psi$ denoting the $A d S_{5}$ Killing spinors

$$
\nabla_{a} \psi=\frac{i}{2} \rho_{a} \psi,
$$

and $\xi$ being further decomposed in terms of two orthogonal unit-norm chiral spinor $\eta_{i}$ [15]

$$
\xi=\sqrt{2} \cos \alpha \eta_{1}+\sqrt{2} \sin \alpha \eta_{2}^{*}
$$

where $\cos 2 \alpha=\sin \zeta$. These two spinors satisfy the following projection conditions

$$
\begin{aligned}
& \gamma^{12} \eta_{1}=-\gamma^{34} \eta_{1}=\gamma^{56} \eta_{1}=i \eta_{1}, \\
& \gamma^{12} \eta_{2}=\gamma^{34} \eta_{2}=\gamma^{56} \eta_{2}=-i \eta_{2}, \\
& \gamma^{3} \eta_{2}^{*}=\eta_{1} .
\end{aligned}
$$

The original geometries preserve eight Killing spinors. In the presence of the deformation to bring the geometry to a Schrödinger invariant form, we discover the additional projection conditions

$$
\begin{aligned}
& \rho^{3} \psi=i \psi, \\
& \Gamma^{-} \eta=0 .
\end{aligned}
$$

With these additional constraints, the spinor $\epsilon_{d}$ satisfies the Killing spinor equations. The kinematical, $\epsilon_{k}$, and superconformal, $\epsilon_{s}$, Killing spinors can then be constructed from the algebra as was illustrated in the earlier text

$$
\begin{aligned}
\epsilon_{k} & =\frac{1}{\sqrt{2}} \Gamma^{+1} \eta \\
\epsilon_{s} & =\left[t-\frac{1}{2} r \Gamma^{+}\left(x_{i} \Gamma^{i}+r \Gamma^{3}\right)\right] \epsilon_{d} .
\end{aligned}
$$




\section{Discussion}

We saw in section 2 that the anti-commutations of two spectator supercharges give the generators for $S U(2)_{1} \times S U(2)_{2}$ as well as the central element $M$.

$$
\left\{\bar{q}^{a \dot{a}}, q_{b \dot{b}}\right\}=\frac{1}{2} \delta_{b}^{a} \delta_{\dot{b}}^{\dot{a}} M-\delta_{b}^{a} R_{\dot{b}}^{\dot{a}}+\delta_{\dot{b}}^{\dot{a}} R_{b}^{a},
$$

From the geometric point of view, the spinor bi-linears $\bar{\epsilon} \Gamma^{m} \epsilon$ made of the Killing spinors $\epsilon_{a \dot{a}}$ corresponding to $q_{a \dot{a}}$ should produce the Killing vectors for the generators on the right hand side of (6.1). Now, recall that $q_{a \dot{\alpha}}$ commute with $(H, D, C, P, \bar{P}, M)$. Inspecting the spinorial Lie derivatives (4.7), especially $\mathfrak{L}_{C} \epsilon$, we find that $\epsilon_{a \dot{a}}$ must be annihilated by $\Gamma^{+}$. This implies that all bi-linears constructed from $\epsilon_{a \dot{a}}$ can have non-zero components only in the $\left(x^{-}\right)$-directions much like the kinematical supercharges $(q, \bar{q})$ discussed earlier:

$$
\left.\bar{\epsilon}^{a \dot{a}} \Gamma^{m} \epsilon_{b \dot{b}}=0 \quad \text { (except for } m=-\right) \text {. }
$$

In particular, the generators for $S U(2)_{1} \times S U(2)_{2}$ symmetry cannot be produced by the Killing spinors. We thus proved without much computation that the Killing spinors for the spectator supercharges with desired algebraic property do not exist within our ansatz.

Even if we give up the $S U(2)_{1} \times S U(2)_{2}$ generators in (6.1), it is still impossible to obtain eight extra Killing spinors as one can see from the following counting argument. We argued above for the projection condition $\Gamma^{+} \epsilon_{a \dot{a}}=0$. The fact that $q_{a \dot{a}}$ transform in the same way under the two $S U(2)$ groups imply that $\partial_{v} \epsilon_{a \dot{a}}=0$, which together with $\mathfrak{L}_{M} \epsilon_{a \dot{a}}=$ 0 yield another projection condition, $\Gamma^{3456} \epsilon_{a \dot{a}}=-\epsilon_{a \dot{a}}$. Finally, since $\epsilon_{a \dot{a}}$ are null Killing spinors, the results of [29] enforces yet another condition, $\Gamma^{9} \epsilon_{a \dot{a}}=\epsilon_{a \dot{a}}$. Three mutually orthogonal projection conditions leave at most $32 / 2^{3}=4$ independent components, so the possiblity of eight extra spinors is excluded.

We have shown that a supergravity background dual to the NR-ABJM theory preserving the super-Schrödinger symmetry and all the global symmetries does not exist. We do not have a clear physical understanding of why this is the case. We end this paper with two possible directions we may pursue to find an explanation. ${ }^{6}$

First, it is conceivable that the singularity problem of the unpolarized BW/LLM solution mentioned in section 2 is unavoidable, so that even if we find a good way to take the non-relativistic limit, the resulting geometry would be necessarily singular. If this is true, we may need to doubt either the existence of the NR-ABJM theory as a quantum field theory or the validity of non-relativistic holography.

Second, note that we have searched for a gravity solution preserving all Schrödinger and global symmetries apart from the non-zero particle number ( $M$-eigenvalue). Via holography, it would correspond to a ground state of the NR-ABJM theory for a fixed non-zero particle number that preserves all the symmetries. It is not obvious a priori whether such a ground state should exist in the field theory. If holography works, the

\footnotetext{
${ }^{6}$ We thank Seok Kim for discussions on the second possibility.
} 
non-existence of the fully symmetric gravity solution may be an indication that the ground states of the field theory necessarily break some parts of the symmetries. It would be interesting to test this idea by studying the spectrum of the field theory directly.

\section{Acknowledgments}

We are grateful for Oren Bergman, Seok Kim, Ki-Myeong Lee and Sungjay Lee for discussions. SL is grateful to the Aspen Center for Physics for hospitality, where parts of this work were carried out. The work of JJ, HK and SL is supported in part by the National Research Foundation of Korea (NRF) Grants No. 2007-331-C00073, 2009-0072755 and 2009-0084601. The work of SL is also supported in part by the NRF Grant No. 20050049409 through the Center for Quantum Spacetime (CQUeST) of Sogang University. 


\section{Appendix}

\section{A. Notations and Conventions}

11-dimensional supergravity The bosonic part of the Lagrangian is

$$
2 \kappa_{11}^{2} \mathcal{L}=R * 1-\frac{1}{2} F \wedge * F-\frac{1}{6} A \wedge F \wedge F .
$$

The fermionic part of the SUSY transformation rule becomes

$$
\delta \psi_{M}=\nabla_{M} \epsilon+\frac{1}{12 \cdot 4 !} F_{I J K L}\left(F_{M}^{I J K L}-8 \delta_{M}^{I} \Gamma^{J K L}\right) \epsilon .
$$

Euler-angle coordinates We take the metric of the $\mathbb{R}^{4}$ to be

$$
d s^{2}=d r^{2}+\frac{r^{2}}{4}\left(\sigma_{1}^{2}+\sigma_{2}^{2}+\sigma_{3}^{2}\right)
$$

where the left-invariant one-forms are defined in terms of Euler angle coordinates by

$$
\begin{aligned}
& \sigma_{1}=+\sin \psi d \theta+\cos \psi \sin \theta d \phi, \\
& \sigma_{2}=-\cos \psi d \theta+\sin \psi \sin \theta d \phi, \\
& \sigma_{3}=d \psi-\cos \theta d \phi .
\end{aligned}
$$

The $S U(2)_{L}$ action is generated by the Killing vectors,

$$
\begin{aligned}
& V_{1}=+\sin \phi \partial_{\theta}+\cot \theta \cos \phi \partial_{\phi}+\csc \theta \cos \phi \partial_{\psi}, \\
& V_{2}=-\cos \phi \partial_{\theta}+\cot \theta \sin \phi \partial_{\phi}+\csc \theta \sin \phi \partial_{\psi}, \\
& V_{3}=-\partial_{\phi},
\end{aligned}
$$

while the $S U(2)_{R}$ action is generated by

$$
\begin{aligned}
& \widehat{V}_{1}=-\sin \psi \partial_{\theta}-\cot \theta \cos \psi \partial_{\psi}-\csc \theta \cos \psi \partial_{\phi}, \\
& \widehat{V}_{2}=+\cos \psi \partial_{\theta}-\cot \theta \sin \psi \partial_{\psi}-\csc \theta \sin \psi \partial_{\phi}, \\
& \widehat{V}_{3}=-\partial_{\psi} .
\end{aligned}
$$

They satisfy the following relations,

$$
\begin{aligned}
& {\left[V_{A}, V_{B}\right]=\epsilon_{A B C} V_{C}, \quad\left[\widehat{V}_{A}, \widehat{V}_{B}\right]=\epsilon_{A B C} \widehat{V}_{C},} \\
& d \sigma_{A}=\frac{1}{2} \epsilon_{A B C} \sigma_{B} \wedge \sigma_{C}, \quad \mathcal{L}_{V_{A}} \sigma_{B}=0, \quad \mathcal{L}_{\widehat{V}_{A}} \sigma_{B}=\epsilon_{A B C} \sigma_{C} .
\end{aligned}
$$

\section{B. Bena-Warner/Lin-Lunin-Maldacena solution}

Review of LLM The geometry is specified by a function $z(x, y)$ defined on the upper half plane $(y \geq 0)$. The function $z$ satisfies the differential equation

$$
\partial_{x}^{2} z+y \partial_{y}\left(y^{-1} \partial_{y} z\right)=0
$$


with a boundary condition at $y=0$. Regularity of the geometry requires that $z= \pm 1 / 2$ on the boundary. It is useful to introduce a few additional variables,

$$
\begin{aligned}
V & : \partial_{x} z=-y \partial_{y} V, \partial_{y} z=y \partial_{x} V \\
G & : z=\frac{1}{2} \tanh G \\
h & : h^{-2}=2 y \cosh G \\
H & : H=h^{2}-h^{-2} V^{2}
\end{aligned}
$$

In terms of these variables, the most general supergravity solution with sixteen supercharges and $S O(1,2) \times S O(4) \times S O(4)$ isometry can be written as

$$
\begin{aligned}
d s^{2}= & H^{-2 / 3}\left(-d t^{2}+d w_{1}^{2}+d w_{2}^{2}\right)+H^{1 / 3}\left[h^{2}\left(d y^{2}+d x^{2}\right)+y e^{G} d \Omega_{3}^{2}+y e^{-G} d \tilde{\Omega}_{3}^{2}\right] \\
F= & -d\left(H^{-1} h^{-2} V\right) \wedge d t \wedge d w_{1} \wedge d w_{2} \\
& -\frac{1}{4} H\left[e^{-3 G} *_{2} d\left(y^{2} e^{2 G}\right) \wedge d \tilde{\Omega}_{3}+e^{3 G} *_{2} d\left(y^{2} e^{-2 G}\right) \wedge d \Omega_{3}\right]
\end{aligned}
$$

Here, $*_{2}$ is the flat epsilon symbol in the $(x, y)$ plane.

Mass deformed $\mathrm{AdS}_{4}$ (without polarization) Section 4.2 of Bena-Warner gives a solution describing the flux deformation of $\mathrm{AdS}_{4} \times S^{7}$. It is instructive to rewrite the solution in the LLM coordinates. How to translate between the two coordinates is explained below.

The result is

$$
\begin{aligned}
z & =\frac{x}{2 \sqrt{x^{2}+y^{2}}}\left[1-\frac{3 \gamma^{2} y^{2}}{\left(x^{2}+y^{2}\right)^{2}}\right], \\
V & =\frac{1}{2 \sqrt{x^{2}+y^{2}}}\left[1+\frac{\gamma^{2}\left(2 x^{2}-y^{2}\right)}{\left(x^{2}+y^{2}\right)^{2}}\right] \\
h^{2} & =\frac{1}{2 \sqrt{x^{2}+y^{2}}}\left[1+\frac{6 \gamma^{2} x^{2}}{\left(x^{2}+y^{2}\right)^{2}}-\frac{9 \gamma^{4} x^{2} y^{2}}{\left(x^{2}+y^{2}\right)^{4}}\right]^{1 / 2}, \\
H & =\frac{\gamma^{2}}{\left(x^{2}+y^{2}\right)^{3 / 2}}\left[1-\frac{\gamma^{2}\left(4 x^{2}+y^{2}\right)}{2\left(x^{2}+y^{2}\right)^{2}}\right]\left[1+\frac{6 \gamma^{2} x^{2}}{\left(x^{2}+y^{2}\right)^{2}}-\frac{9 \gamma^{4} x^{2} y^{2}}{\left(x^{2}+y^{2}\right)^{4}}\right]^{-1 / 2} .
\end{aligned}
$$

The parameter $\gamma$ is related to those of BW [7] by

$$
\gamma^{2}=\left(128 L^{6} \beta^{2} R^{6}\right)_{B W}
$$

In the UV region $(x, y \gg \gamma)$, all the square brackets in (B.7)-B.10) can be ignored, and we recover the $\mathrm{AdS}_{4} \times S^{7}$ geometry upon a suitable constant rescaling of coordinates.

Relation between Bena-Warner and Lin-Lunin-Maldacena We compare the notations of Bena and Warner (BW) [7] and those of Lin, Lunin and Maldacena (LLM) [8]. This was already done in appendix $\mathrm{C}$ of [8], but it contained some minor errors. 
The BW and LLM metrics read,

$$
\begin{aligned}
d s_{B W}^{2}= & 16 L^{4} e^{2 B_{0}}\left(-d t^{2}+d w_{1}^{2}+d w_{2}^{2}\right)+e^{2 B_{1}-B_{0}}\left(d u^{2}+d v^{2}\right) \\
& +u^{2} e^{2 B_{3}-B_{0}} d \Omega_{3}^{2}+v^{2} e^{-2 B_{3}-B_{0}} d \widetilde{\Omega}_{3}^{2} \\
d s_{L L M}^{2}= & H^{-2 / 3}\left(-d t^{2}+d w_{1}^{2}+d w_{2}^{2}\right) \\
& +H^{1 / 3}\left[h^{2}\left(d y^{2}+d x^{2}\right)+y e^{G} d \Omega_{3}^{2}+y e^{-G} d \tilde{\Omega}_{3}^{2}\right],
\end{aligned}
$$

which lead to the identifications,

$$
\begin{aligned}
& H^{-2 / 3}=16 L^{4} e^{2 B_{0}}, y e^{G}=4 L^{2} u^{2} e^{2 B_{3}}, y e^{-G}=4 L^{2} e^{-2 B_{3}}, \\
& h^{2}\left(d x^{2}+d y^{2}\right)=4 L^{2} e^{2 B_{1}}\left(d u^{2}+d v^{2}\right) .
\end{aligned}
$$

Combining the $G-B_{3}$ relations, we find

$$
e^{G}=\frac{u}{v} e^{2 B_{3}}, \quad y=4 L^{2} u v .
$$

Orthogonality of the coordinates implies

$$
x=2 L^{2}\left(u^{2}-v^{2}\right) .
$$

Putting $(\overline{B .15})$ and $(\overline{B .16})$ back to $(\overline{B .14})$, we find

$$
h^{-2}=4 L^{2} e^{-2 B_{1}}\left(u^{2}+v^{2}\right) .
$$

As a cross check, we note that inserting (B.15) and (B.17) into the LLM relation (B.4),

$$
h^{-2}=y\left(e^{G}+e^{-G}\right),
$$

reproduces eq. (43) of [7],

$$
e^{-2 B_{1}}\left(u^{2}+v^{2}\right)=u^{2} e^{2 B_{3}}+v^{2} e^{-2 B_{3}} .
$$

In translating the BW solution into the LLM form, it is most convenient to use first

$$
2 z=\frac{e^{2 G}-1}{e^{2 G}+1}=\frac{u^{2} e^{4 B_{3}}-v^{2}}{u^{2} e^{4 B_{3}}+v^{2}},
$$

and then use the LLM formulas to compute other quantities. 


\section{References}

[1] D. T. Son, "Toward an AdS/cold atoms correspondence: a geometric realization of the Schroedinger symmetry," Phys. Rev. D 78, 046003 (2008) [arXiv:0804.3972 [hep-th]].

[2] K. Balasubramanian and J. McGreevy, "Gravity duals for non-relativistic CFTs," Phys. Rev. Lett. 101, 061601 (2008) [arXiv:0804.4053 [hep-th]].

[3] Y. Nakayama, M. Sakaguchi and K. Yoshida, "Non-Relativistic M2-brane Gauge Theory and New Superconformal Algebra," JHEP 0904, 096 (2009) [arXiv:0902.2204 [hep-th]].

[4] K. M. Lee, S. Lee and S. Lee, "Nonrelativistic Superconformal M2-Brane Theory," JHEP 0909, 030 (2009) [arXiv:0902.3857 [hep-th]].

[5] O. Aharony, O. Bergman, D. L. Jafferis and J. Maldacena, 'N=6 superconformal Chern-Simons-matter theories, M2-branes and their gravity duals," JHEP 0810, 091 (2008) [arXiv:0806.1218 [hep-th]].

[6] I. Bena, 'The M-theory dual of a 3 dimensional theory with reduced supersymmetry," Phys. Rev. D 62, 126006 (2000) [arXiv:hep-th/0004142].

[7] I. Bena and N. P. Warner, "A harmonic family of dielectric flow solutions with maximal supersymmetry," JHEP 0412, 021 (2004) [arXiv:hep-th/0406145].

[8] H. Lin, O. Lunin and J. M. Maldacena, "Bubbling AdS space and 1/2 BPS geometries," JHEP 0410, 025 (2004) [arXiv:hep-th/0409174].

[9] K. Hosomichi, K. M. Lee, S. Lee, S. Lee and J. Park, "N=5,6 Superconformal Chern-Simons Theories and M2-branes on Orbifolds," JHEP 0809, 002 (2008) [arXiv:0806.4977 [hep-th]].

[10] J. Gomis, D. Rodriguez-Gomez, M. Van Raamsdonk and H. Verlinde, "A Massive Study of M2-brane Proposals," JHEP 0809, 113 (2008) [arXiv:0807.1074 [hep-th]].

[11] C. Duval and P. A. Horvathy, “On Schrodinger superalgebras,” J. Math. Phys. 35, 2516 (1994) [arXiv:hep-th/0508079].

[12] M. Leblanc, G. Lozano and H. Min, "Extended superconformal Galilean symmetry in Chern-Simons matter systems," Annals Phys. 219, 328 (1992) [arXiv:hep-th/9206039].

[13] A. Galajinsky and I. Masterov, "Remark on quantum mechanics with N=2 superconformal Galilean symmetry," arXiv:0902.2910 [hep-th].

[14] H. Ooguri and C. S. Park, "Supersymmetric non-relativistic geometries in M-theory," Nucl. Phys. B 824, 136 (2010) [arXiv:0905.1954 [hep-th]].

[15] J. P. Gauntlett, D. Martelli, J. Sparks and D. Waldram, "Supersymmetric AdS(5) solutions of M-theory," Class. Quant. Grav. 21, 4335 (2004) [arXiv:hep-th/0402153].

[16] E. O. Colgain and H. Yavartanoo, “NR CFT 3 duals in M-theory,” JHEP 0909, 002 (2009) [arXiv:0904.0588 [hep-th]].

[17] Y. Nakayama and S. J. Rey, "Observables and Correlators in Nonrelativistic ABJM Theory," JHEP 0908, 029 (2009) [arXiv:0905.2940 [hep-th]].

[18] N. Bobev, A. Kundo and K. Pilch, "Supersymmetric IIB Solutions with Schrödinger symmetry," JHEP 0907, 107 (2009) [arXiv:0905.0673 [hep-th]].

[19] A. Donos and J. P. Gauntlett, "Schrodinger invariant solutions of type IIB with enhanced supersymmetry," arXiv:0907.1761 [hep-th]. 
[20] C. P. Herzog, M. Rangamani and S. F. Ross, "Heating up Galilean holography," JHEP 0811, 080 (2008) [arXiv:0807.1099 [hep-th]].

[21] J. Maldacena, D. Martelli and Y. Tachikawa, "Comments on string theory backgrounds with non-relativistic conformal symmetry," JHEP 0810, 072 (2008) [arXiv:0807.1100 [hep-th]].

[22] A. Adams, K. Balasubramanian and J. McGreevy, "Hot Spacetimes for Cold Atoms," JHEP 0811, 059 (2008) [arXiv:0807.1111 [hep-th]].

[23] J. P. Gauntlett, S. Kim, O. Varela and D. Waldram, “Consistent supersymmetric Kaluza-Klein truncations with massive modes," JHEP 0904, 102 (2009) [arXiv:0901.0676 [hep-th]].

[24] E. O Colgain, O. Varela and H. Yavartanoo, “Non-relativistic M-Theory solutions based on Kaehler-Einstein spaces," JHEP 0907, 081 (2009) [arXiv:0906.0261 [hep-th]].

[25] Y. Nishida and D. T. Son, "Nonrelativistic conformal field theories," Phys. Rev. D 76, 086004 (2007) [arXiv:0706.3746 [hep-th]].

[26] M. Blau, J. Hartong and B. Rollier, "Geometry of Schroedinger Space-Times, Global Coordinates, and Harmonic Trapping," JHEP 0907, 027 (2009) [arXiv:0904.3304 [hep-th]].

[27] J. M. Figueroa-O'Farrill, “On the supersymmetries of anti de Sitter vacua," Class. Quant. Grav. 16, 2043 (1999) [arXiv:hep-th/9902066].

[28] J. P. Gauntlett and S. Pakis, “The geometry of D = 11 Killing spinors," JHEP 0304, 039 (2003) [arXiv:hep-th/0212008].

[29] J. P. Gauntlett, J. B. Gutowski and S. Pakis, "The geometry of D = 11 null Killing spinors," JHEP 0312, 049 (2003) [arXiv:hep-th/0311112].

[30] A. Donos and J. P. Gauntlett, "Solutions of type IIB and D=11 supergravity with Schrodinger(z) symmetry," JHEP 0907, 042 (2009) [arXiv:0905.1098 [hep-th]].

[31] A. Donos and J. P. Gauntlett, "Supersymmetric solutions for non-relativistic holography," JHEP 0903, 138 (2009) [arXiv:0901.0818 [hep-th]].

[32] S. A. Hartnoll and K. Yoshida, "Families of IIB duals for nonrelativistic CFTs," JHEP 0812, 071 (2008) [arXiv:0810.0298 [hep-th]].

[33] J. P. Gauntlett, D. Martelli, J. Sparks and D. Waldram, "Sasaki-Einstein Metrics on $S^{2} \times S^{3}$," Adv. Theor. Math. Phys. 8, 711-734 (2004) [arXiv:hep-th/0403002]. 